\title{
Stationary Navier-Stokes Flow Around a Rotating Obstacle
}

\author{
By \\ Reinhard FARwig and Toshiaki HishIDA \\ (Technische Universität Darmstadt, Germany and Niigata University, Japan) \\ Dedicated to Professor Tetsuro Miyakawa on his 60th birthday
}

\begin{abstract}
Consider a viscous incompressible fluid filling the whole 3-dimensional space exterior to a rotating body with constant angular velocity $\omega$. By using a coordinate system attached to the body, the problem is reduced to an equivalent one in a fixed exterior domain. The reduced equation involves the crucial drift operator $(\omega \wedge x) \cdot \nabla$, which is not subordinate to the usual Stokes operator. This paper addresses stationary flows to the reduced problem with an external force $f=\operatorname{div} F$, that is, time-periodic flows to the original one. Generalizing previous results of G. P. Galdi [19] we show the existence of a unique solution $(\nabla u, p)$ in the class $L_{3 / 2, \infty}$ when both $F \in L_{3 / 2, \infty}$ and $\omega$ are small enough; here $L_{3 / 2, \infty}$ is the weak- $L_{3 / 2}$ space.

Key Words and Phrases. Navier-Stokes flow, Rotating obstacle, Exterior domain, Weak stationary solutions, Weak- $L_{p}$ spaces.

2000 Mathematics Subject Classification Numbers. 35Q30, 76D05.
\end{abstract}

\section{Introduction}

Let $D$ be an exterior domain in $\boldsymbol{R}^{3}$ with smooth boundary $\partial D$. We consider the motion of a viscous incompressible fluid filling the domain $D$ when the obstacle $R^{3} \backslash D$, which consists of a finite number of rigid bodies, is rotating about an axis with constant angular velocity $\omega$. Without loss of generality, we may assume that $\omega=|\omega| e_{3}=(0,0,|\omega|)^{T}$. The fluid at space infinity is assumed to be at rest. Our aim is to solve the Navier-Stokes system in the timedependent domain

$$
D(t)=\{y=O(|\omega| t) x ; x \in D\}, \quad \text { where } O(t)=\left(\begin{array}{ccc}
\cos t & -\sin t & 0 \\
\sin t & \cos t & 0 \\
0 & 0 & 1
\end{array}\right)
$$

subject to the non-slip boundary condition on the surface $\partial D(t)$, so that the fluid velocity attains $\omega \wedge y$ there. Here, $\wedge$ denotes the usual exterior product of three-dimensional vectors; thus,

$$
\omega \wedge y=|\omega|\left(-y_{2}, y_{1}, 0\right)^{T}=\frac{d}{d t} O(|\omega| t) x
$$


which describes the velocity at a point $y$ of the rotating rigid body. By using a coordinate system attached to the obstacle (see [25]), one can reduce the problem to an equivalent one in the fixed exterior domain $D$; that is,

$$
\begin{aligned}
\frac{\partial u}{\partial t}+u \cdot \nabla u & =\Delta u+(\omega \wedge x) \cdot \nabla u-\omega \wedge u-\nabla p+f \\
\operatorname{div} u & =0
\end{aligned}
$$

in $D \times(0, \infty)$ subject to

$$
\left.u\right|_{\partial D}=\omega \wedge x, \quad u \rightarrow 0 \quad \text { as }|x| \rightarrow \infty,\left.\quad u\right|_{t=0}=a,
$$

where $u=\left(u_{1}, u_{2}, u_{3}\right)^{T}$ and $p$ are the unknown velocity and pressure of the fluid, respectively, while the external force $f$ and initial velocity $a$ are prescribed.

A significant feature which distinguishes the problem above from the usual Navier-Stokes system $(\omega=0)$ is the presence of the first order term with unbounded coefficients, viz.

$$
(\omega \wedge x) \cdot \nabla u=|\omega|\left(-x_{2} \frac{\partial u}{\partial x_{1}}+x_{1} \frac{\partial u}{\partial x_{2}}\right)=|\omega| \frac{\partial u}{\partial \theta},
$$

where, in the final representation, $\theta=\tan ^{-1}\left(x_{2} / x_{1}\right)$ is the angular variable in a cylindrical coordinate system. Even though $|\omega|>0$ is small, this term is not subordinate to the viscous term $\Delta u$ in the sense that some properties of the linear operator

$$
L=-\Delta-(\omega \wedge x) \cdot \nabla+\omega \wedge
$$

are worse than those of the Laplacian. In fact, by [25], we know that the generated semigroup $e^{-t L}$ for the whole space problem does not map $L_{2}\left(\boldsymbol{R}^{3}\right)$ into the domain $D(L)$ for $t>0$, and that

$$
\sigma(-L) \supseteqq \sigma_{\mathrm{ess}}(-L)=\{\lambda=\alpha+i k|\omega| \in \boldsymbol{C} ; \alpha \leq 0, k \in \boldsymbol{Z}\},
$$

see [16] for the operator $-P L$ where $P$ is the Helmholtz projection on $L_{2}(D)$. Hence $e^{-t L}$ is not an analytic semigroup. Moreover, by [14], the fundamental solution $\Gamma(x, y)$ of the operator $L$ cannot be dominated by $|x-y|^{-1}$; more precisely, its component $\Gamma_{33}(x, y)$ satisfies

$$
\Gamma_{33}(x, y) \geq C \frac{\log |x-y|}{|x-y|}
$$

when, for example, $x=\rho e_{1}$ and $y=\rho e_{2}$ with large $\rho>0$. These observations tell us that the operator $L$ cannot be treated by means of any perturbation argument and could cause some mathematical difficulties. 
In the last decade a lot of effort has gone into analyzing nonstationary as well as stationary problems for the nonlinear and linearized system in either an exterior domain or the whole space; moreover, $L_{2}$ theory and $L_{q}$ theory were used for problems without as well as with translation of the obstacle. We refer to [3], [9], [10], [12], [13], [14], [15], [16], [18], [19], [20], [21], [23], [24], [25], [26], [27], [28], [33], [34] and [36]. Nevertheless, our mathematical understanding is still far from complete. There are also some other studies, to which we don't refer here, such as moving bodies in a bounded or unbounded fluid region, rotating fluids without bodies in the whole space, etc.

The present paper is devoted to the study of the nonlinear stationary problem in exterior domains:

$$
L u+\nabla p+u \cdot \nabla u=f, \quad \operatorname{div} u=0 \quad \text { in } D
$$

subject to

$$
\left.u\right|_{\partial D}=\omega \wedge x, \quad u \rightarrow 0 \quad \text { as }|x| \rightarrow \infty .
$$

Note that the stationary motion in the frame attached to the obstacle corresponds to a time-periodic one in the original frame; in fact, given a solution $(u, p)$ of (1.2), (1.3), the pair of $O(|\omega| t) u\left(O(|\omega| t)^{T} x\right)$ and $p\left(O(|\omega| t)^{T} x\right)$ provides a periodic solution of the original problem in the domain $D(t)$ with external force $O(|\omega| t) f\left(O(|\omega| t)^{T} x\right)$. It is possible to construct solutions of class $\nabla u \in L_{2}$ to (1.2), (1.3) by means of the Galerkin method in the $L_{2}$ framework for arbitrary $\omega$ and $f=\operatorname{div} F, F \in L_{2}$, see Borchers [3], Galdi [18], Serre [34] and Silvestre [36]. When $\omega$ is small enough and $f=\operatorname{div} F$ satisfies the decay estimates

$$
|x|^{2}|F(x)|+|x|^{3}|f(x)|+|x|^{4}|\operatorname{div} f(x)| \leq c_{0}
$$

for some small $c_{0}>0$, Galdi [19] derived the pointwise estimates

$$
|x||u(x)|+|x|^{2}(|\nabla u(x)|+|p(x)|)+|x|^{3}|\nabla p(x)| \leq C
$$

of a unique solution. These decay properties are important in the study of stability ([3], [20], [29]), but, at first glance, rather surprising in view of (1.1). In [21] Galdi and Silvestre have recently extended a part of [19]; that is, they have shown $|x||u(x)| \leq C$ for a small force $f=\operatorname{div} F \in L_{2}$ with $|x|^{2}|F(x)| \leq c_{0}$ when the translation of the obstacle is also taken into account. We may expect an anisotropic decay structure of solutions similar to the Oseen case ([11], [17]), but as far as simple isotropic decay estimates are concerned, the result of Galdi [19] shows that the rate of decay of Navier-Stokes flow at infinity is the same as in the usual case $\omega=0$ in spite of the slightly worse behavior (1.1) of the fundamental solution. 
The purpose of the present paper is to provide another outlook on the pointwise estimate (1.4) in a different framework by use of special function spaces. To be more precise, we show in the class $L_{3 / 2, \infty}$ the existence of a unique solution $(\nabla u, p)$ to $(1.2),(1.3)$ with force $f \in \dot{W}_{3 / 2, \infty}^{-1}$ when both $f$ and $\omega$ are small enough; here $L_{3 / 2, \infty}$ is the weak- $L_{3 / 2}$ space, one of the Lorentz spaces. We note that $f \in \dot{W}_{3 / 2, \infty}^{-1}$ if and only if $f=\operatorname{div} F$ with $F \in L_{3 / 2, \infty}$, see Lemma 2.2 (i) below. For more precise definitions, in particular of weak solutions to (1.2), (1.3), see Section 2.

Now our main theorem reads as follows.

Theorem 1.1. There is a constant $\eta=\eta(D)>0$ such that for

$$
f \in \dot{W}_{3 / 2, \infty}^{-1}(D)
$$

with

$$
|\omega|+\|f\|_{\dot{W}_{3 / 2, \infty}^{-1}(D)} \leq \eta,
$$

problem (1.2), (1.3) possesses a unique weak solution $(u, p)$ with

$$
\nabla u, p \in L_{3 / 2, \infty}(D)
$$

subject to the estimate

$$
\|\nabla u\|_{3 / 2, \infty}+\|u\|_{3, \infty}+\|p\|_{3 / 2, \infty} \leq C\left(|\omega|+\|f\|_{\dot{W}_{3 / 2, \infty}^{-1}(D)}\right),
$$

with some $C>0$ independent of $|\omega|$ and $f$.

Our class of solutions is consistent with (1.4), and our class of external forces is larger than in [19], [21], though one cannot expect explicit pointwise estimates as in (1.4) for such external forces. For the case $\omega=0$, a result analogous to Theorem 1.1 has been proved by Kozono and Yamazaki [32]. Further regularity of the solution obtained in Theorem 1.1 under an additional assumption on the external force is discussed in Section 6, see Theorem 6.1.

In [28], based on an idea from [14], one of the present authors has established the existence, uniqueness and $L_{q}$ estimate

$$
\|\nabla u\|_{q}+\|p\|_{q} \leq C\|f\|_{\dot{W}_{q}^{-1}(D)}
$$

of weak solutions to the linearized problem

$$
L u+\nabla p=f, \quad \operatorname{div} u=0 \quad \text { in } D ;\left.\quad u\right|_{\partial D}=0,
$$

provided that $(n /(n-1)=) 3 / 2<q<3(=n)$. This result is regarded as a generalization of [4], [22], [30], [31] in the usual case $\omega=0$, since the restriction on the exponent $q$ is the same. Since the case $q=3 / 2(=n / 2)$ needed to estimate the nonlinearity $u \cdot \nabla u$ is missing in [28], an $L_{q}$ theory does not help to 
solve the nonlinear problem (1.2), (1.3). Note that $L_{3 / 2}$ is too restrictive at infinity to expect $\nabla u \in L_{3 / 2}$ for (1.8) even if $f=\operatorname{div} F$ with $F \in C_{0}^{\infty}$. Therefore, we have to replace $L_{3 / 2}$ by a larger space. To do so, we follow Kozono and Yamazaki [32] who for the first time used Lorentz spaces in the case $\omega=0$. This paper shows that the right class to find a solution $(\nabla u, p)$ to $(1.2),(1.3)$ is $L_{3 / 2, \infty}$ as well.

An important step is to derive, instead of (1.7), the a priori estimate

$$
\|\nabla u\|_{3 / 2, \infty}+\|p\|_{3 / 2, \infty} \leq C\|f\|_{\dot{W}_{3 / 2, \infty}^{-1}(D)}
$$

for the linearized problem (1.8); in fact, once this is established, a fixed point argument yields a unique solution $(\nabla u, p)$ of $(1.2),(1.3)$ in the class $L_{3 / 2, \infty}$. In the proof of the solvability of (1.8) for all $f \in \dot{W}_{3 / 2, \infty}^{-1}$, a duality argument due to Kozono and Yamazaki [32] does not seem to be applicable to our problem because of lack of homogeneity of the equation, unlike the usual case $\omega=0$. We thus follow, in principle, the argument of Shibata and Yamazaki [35], in which the solution is constructed without any duality argument for the Oseen problem. Note that one cannot use any continuity argument since $C_{0}^{\infty}$ is not dense in $L_{q, \infty}$. So, as in [35], given $f \in \dot{W}_{3 / 2, \infty}^{-1}$, we try to construct directly the solution to (1.8). Though cut-off procedures were carried out twice in [35], we use such a procedure only once to obtain the solution; in this point, the proof of [35] is simplified in the present paper. In fact, a parametrix $(v, \pi)$, an approximation of the solution, constructed by use of solutions in the whole space and in a bounded domain combined with the Bogovskii operator [2] satisfies

$$
L v+\nabla \pi=f+R f, \quad \operatorname{div} v=0 \quad \text { in } D ;\left.\quad v\right|_{\partial D}=0,
$$

where $R f$ is a remainder term with compact support. We show that the operator $1+R$ has a bounded inverse in $\dot{W}_{3 / 2, \infty}^{-1}$. In the proof, the embedding $\dot{W}_{3,1}^{1} \hookrightarrow L_{\infty}\left(\left[32\right.\right.$, Lemma 2.1]) and the fact that the dual space of $\dot{W}_{3,1}^{1}$ is $\dot{W}_{3 / 2, \infty}^{-1}$ play a fundamental role.

Besides Theorem 1.1 for the nonlinear problem (1.2), (1.3), in order to make the linear theory complete, we consider existence, uniqueness and a priori estimates of solutions to the linearized problem (1.8) with an external force $f=\operatorname{div} F, F \in L_{q, r}$, where $L_{q, r}$ is the general Lorentz space introduced in Section 2. This study supplements the important case $(q, r)=(3 / 2, \infty)$ above and the case $q=r \in(3 / 2,3)$ discussed in [28]. It turns out that a unique solution exists if either $1<q<3,1 \leq r \leq \infty$ or $(q, r)=(3,1)$; in fact, the latter case is critical to control the behavior of solutions at infinity and, therefore, to show the uniqueness. From the viewpoint of decay structure of solutions at infinity, our existence result for (1.8) can be divided into two theorems. The first theorem (Theorem 5.1) possesses the usual a priori estimate 


$$
\|\nabla u\|_{q, r}+\|p\|_{q, r} \leq C\|f\|_{\dot{W}_{q, r}^{-1}(D)}
$$

for one of the following cases:

$$
\left\{\begin{array}{l}
(q, r)=(3 / 2, \infty) \\
3 / 2<q<3,1 \leq r \leq \infty, \\
(q, r)=(3,1)
\end{array}\right.
$$

This was first proved by Kozono and Yamazaki [32] for the case $\omega=0$. The second theorem (Theorem 5.2) tells us that the solution $(\nabla u, p)$ decays like $1 /|x|^{2}$ in general for the other cases

$$
\left\{\begin{array}{l}
1<q<3 / 2,1 \leq r \leq \infty \\
q=3 / 2,1 \leq r<\infty
\end{array}\right.
$$

in which it is impossible to show $R f \in \dot{W}_{q, r}^{-1}$ in (1.10), differently from the cases (1.11). To be precise, the solution is decomposed as $u=v+\tilde{v}, p=\pi+\tilde{\pi}$ with a good decay part $(\nabla v, \pi) \in L_{q, r}$ and a critical decay part $(\nabla \tilde{v}, \tilde{\pi}) \in L_{3 / 2, \infty}$. This idea of decomposition was due to Shibata and Yamazaki [35] for the Oseen problem. The first part $(v, \pi)$ is constructed as in the case $(q, r)=(3 / 2, \infty)$ explained above and it satisfies (1.10). Therefore, the second part $(\tilde{v}, \tilde{\pi})$ should obey

$$
L \tilde{v}+\nabla \tilde{\pi}=-R f, \quad \operatorname{div} \tilde{v}=0 \quad \text { in } D ;\left.\quad \tilde{v}\right|_{\partial D}=0 .
$$

Since the support of $R f$ is compact, we see $R f \in \dot{W}_{3 / 2, \infty}^{-1}$ and can apply the result for the case $(q, r)=(3 / 2, \infty)$ to solve (1.13). Consequently our proof is considerably shortened in comparison with [35] in which a parametrix for (1.13) was constructed once more via cut-off procedure and, among other techniques, Proposition 3.3 (without the term $(\omega \wedge x) \cdot \nabla u)$ for the whole space case, see Section 3 below, was important.

We finally mention that the stability of the stationary flow obtained in Theorem 1.1 is studied by [29] within the same framework of Lorentz spaces as adopted in the present paper.

This paper is organized as follows. In the next section we start with some preliminaries. To prove our main theorem on the stationary Navier-Stokes problem (1.2), (1.3) in Section 6, we carry out the analysis of the linearized equation (1.8) in the following order: the whole space problem in Section 3, the interior problem in Section 4 and finally the exterior problem in Section 5.

\section{Preliminaries}

Given any smooth domain $\Omega \subset \boldsymbol{R}^{3}$ such as $\Omega=D, \Omega=\boldsymbol{R}^{3}$ or a bounded domain we introduce the following function spaces. By $C_{0}^{\infty}(\Omega)$ we denote the 
class of smooth functions with compact support in $\Omega$. For $1 \leq q \leq \infty$, the usual Lebesgue spaces are denoted by $L_{q}(\Omega)$ with norm $\|\cdot\|_{q, \Omega}$. We need the Lorentz spaces $L_{q, r}(\Omega)$, with norm $\|\cdot\|_{q, r, \Omega}$ : for $1<q<\infty$ and $1 \leq r \leq \infty$, the Lorentz spaces can be constructed via real interpolation

$$
L_{q, r}(\Omega)=\left(L_{1}(\Omega), L_{\infty}(\Omega)\right)_{1-1 / q, r}
$$

for details, see Bergh and Löfström [1]. Note that

$$
L_{q, r_{0}}(\Omega) \subset L_{q, r_{1}}(\Omega) \quad \text { if } r_{0} \leq r_{1} ; \quad L_{q, q}(\Omega)=L_{q}(\Omega),
$$

and, if $\Omega$ is bounded, that

$$
L_{p, r}(\Omega) \subset L_{q, s}(\Omega) \quad \text { for all } 1<q<p<\infty, r, s \in[1, \infty],
$$

with continuous embeddings. For

$$
1<q<\infty, \quad 1<r \leq \infty, \quad \frac{1}{q^{\prime}}+\frac{1}{q}=1, \quad \frac{1}{r^{\prime}}+\frac{1}{r}=1,
$$

we have the duality relation

$$
L_{q, r}(\Omega)=L_{q^{\prime}, r^{\prime}}(\Omega)^{*} .
$$

In particular, $L_{q, \infty}(\Omega)=L_{q^{\prime}, 1}(\Omega)^{*}$ is well known as the weak- $L_{q}$ space, in which $C_{0}^{\infty}(\Omega)$ is not dense; moreover, $f \in L_{q, \infty}(\Omega)$ if and only if

$$
\sup _{\sigma>0} \sigma|\{x \in \Omega ;|f(x)|>\sigma\}|^{1 / q}<\infty,
$$

where $|\cdot|$ stands for the Lebesgue measure. In what follows, we adopt the same symbols for vector and scalar function spaces as long as there is no confusion, and we use the abbreviations $\|\cdot\|_{q}=\|\cdot\|_{q, D}$ and $\|\cdot\|_{q, r}=\|\cdot\|_{q, r, D}$ for the exterior domain $D$.

Furthermore, we need homogeneous Sobolev spaces. For $1<q<\infty$, let $\dot{W}_{q}^{1}(\Omega)$ be the completion of $C_{0}^{\infty}(\Omega)$ with respect to the norm $\|\nabla(\cdot)\|_{q, \Omega}$, and let $\dot{W}_{q}^{-1}(\Omega)$ be the dual space of $\dot{W}_{q^{\prime}}^{1}(\Omega)$ where $1 / q^{\prime}+1 / q=1$. Let

$$
1<q_{0}<q<q_{1}<\infty, \quad 1 / q=(1-\theta) / q_{0}+\theta / q_{1}, \quad 1 \leq r \leq \infty .
$$

We then define

$$
\dot{W}_{q, r}^{1}(\Omega)=\left(\dot{W}_{q_{0}}^{1}(\Omega), \dot{W}_{q_{1}}^{1}(\Omega)\right)_{\theta, r},
$$

which is independent of the choice of $\left(q_{0}, q_{1}\right)$, with norm $\|\nabla(\cdot)\|_{q, r, \Omega}$. Note that $C_{0}^{\infty}(\Omega)$ is not dense in $\dot{W}_{q, \infty}^{1}(\Omega)$.

For $(q, r)$ satisfying $(2.2)$, the space $\dot{W}_{q, r}^{-1}(\Omega)$ is defined as the dual space of $\dot{W}_{q^{\prime}, r^{\prime}}^{1}(\Omega)$; by the duality theorem for interpolation spaces $([1,3.7 .1])$, we see that 


$$
\dot{W}_{q, r}^{-1}(\Omega)=\left(\dot{W}_{q_{0}}^{-1}(\Omega), \dot{W}_{q_{1}}^{-1}(\Omega)\right)_{\theta, r}
$$

for $q, q_{0}, q_{1}, r$ satisfying (2.3) with $1<r \leq \infty$. For $r=1$, we also define $\dot{W}_{q, 1}^{-1}(\Omega), 1<q<\infty$, as the dual space of the completion of $C_{0}^{\infty}(\Omega)$ with respect to the norm $\|\nabla(\cdot)\|_{q^{\prime}, \infty, \Omega}$, which we denote by $\hat{W}_{q^{\prime}, \infty}^{1}(\Omega)\left(\varsubsetneqq \dot{W}_{q^{\prime}, \infty}^{1}(\Omega)\right)$. Then (2.4) holds for $r=1$ as well, see [1, p. 55].

For later use we cite the following results.

Lemma 2.1. Let $\Omega \subset \boldsymbol{R}^{3}$ be a bounded domain.

(i) The embedddings $\dot{W}_{q, r}^{1}(\Omega) \hookrightarrow L_{q, r}(\Omega), \quad 1<q<\infty, \quad 1 \leq r \leq \infty$, and $L_{q, r}(\Omega) \hookrightarrow \dot{W}_{q, r}^{-1}(\Omega), 1<q<\infty, 1 \leq r \leq \infty$, are compact.

(ii) Let $1<q<\infty$ and $1 \leq r \leq \infty$. Then

$$
\dot{W}_{q, r}^{1}(\Omega)=\left\{u \in L_{q, r}(\Omega) ; \nabla u \in L_{q, r}(\Omega),\left.u\right|_{\partial \Omega}=0\right\},
$$

and for all $u \in \dot{W}_{q, r}^{1}(\Omega)$ holds the Poincaré inequality

$$
\|u\|_{q, r, \Omega} \leq C\|\nabla u\|_{q, r, \Omega} .
$$

Proof. (i) follows from (2.1), classical embedding theorems, duality and interpolation, see $[1,3.14 .8]$. (ii) is based on the classical Poincaré inequality and interpolation.

Lemma 2.2. (i) ([32, Lemma 2.2]). Let $\Omega \subset \boldsymbol{R}^{3}$ be any domain and let $1<q<\infty, 1 \leq r \leq \infty$. Then there exists a constant $C>0$ such that for every $f \in \dot{W}_{q, r}^{-1}(\Omega)$ there is $F \in L_{q, r}(\Omega)$ satisfying

$$
\operatorname{div} F=f, \quad\|F\|_{q, r, \Omega} \leq C\|f\|_{\dot{W}_{q, r}^{-1}(\Omega)} .
$$

(ii) ([32, Lemma 2.1]). Let $D \subset \boldsymbol{R}^{3}$ be an exterior domain. Then for any $1<q<3$ and $r \in[1, \infty]$ we have, with $1 / q_{*}:=1 / q-1 / 3$, the characterization

$$
\dot{W}_{q, r}^{1}(D)=\left\{u \in L_{q_{*}, r}(D) ; \nabla u \in L_{q, r}(D),\left.u\right|_{\partial D}=0\right\},
$$

together with the embedding estimate

$$
\|u\|_{q_{*}, r} \leq C\|\nabla u\|_{q, r} .
$$

(iii) ([32, Lemma 2.1]). Let $D \subset \boldsymbol{R}^{3}$ be an exterior domain. Then for $q=3$ we have the embedding $\dot{W}_{3,1}^{1}(D) \hookrightarrow L_{\infty}(D) \cap C(D)$ with the estimate

$$
\|u\|_{\infty} \leq \frac{1}{3}\|\nabla u\|_{3,1}
$$

and $u(x) \rightarrow 0$ as $|x| \rightarrow \infty$ for $u \in \dot{W}_{3,1}^{1}(D)$.

(iv) ([5, Theorem 5.9]). Let $D \subset \boldsymbol{R}^{3}$ be an exterior domain, let $1<q<3$, $r \in[1, \infty]$, and let $u \in L_{1, \mathrm{loc}}(\bar{D})$ satisfy $\nabla u \in L_{q, r}(D)$. Then there is a constant $k=k(u)$ such that $u+k \in L_{q_{*}, r}(D)$ and 


$$
\|u+k\|_{q_{*}, r} \leq C\|\nabla u\|_{q, r},
$$

with some $C>0$ independent of $u$; here $1 / q_{*}=1 / q-1 / 3$.

For given $1<q<\infty, 1 \leq r \leq \infty$ and $f \in \dot{W}_{q, r}^{-1}(D)$ let us first consider the boundary value problem for the linearized equation

$$
\begin{cases}-\Delta u-(\omega \wedge x) \cdot \nabla u+\omega \wedge u+\nabla p=f & \text { in } D \\ \operatorname{div} u=0 & \text { in } D, \\ u=0 & \text { on } \partial D\end{cases}
$$

Then the pair of functions $(u, p)$ is called $(q, r)$-weak solution ( $q$-weak solution when $q=r$ ) of (2.9) if

1. $(u, p) \in \dot{W}_{q, r}^{1}(D) \times L_{q, r}(D)$;

2. $\operatorname{div} u=0$ in $L_{q, r}(D)$;

3. $(\omega \wedge x) \cdot \nabla u-\omega \wedge u \in \dot{W}_{q, r}^{-1}(D)$;

4. $(u, p)$ satisfies $(2.9)_{1}$ in the sense of distributions, that is,

$$
\langle\nabla u, \nabla \varphi\rangle-\langle(\omega \wedge x) \cdot \nabla u-\omega \wedge u, \varphi\rangle-\langle p, \operatorname{div} \varphi\rangle=\langle f, \varphi\rangle
$$

holds for all $\varphi \in C_{0}^{\infty}(D)$, where $\langle\cdot, \cdot\rangle$ stands for various duality pairings.

By continuity, $(u, p)$ satisfies (2.10) for all $\varphi \in \dot{W}_{q^{\prime}, r^{\prime}}^{1}(D)$ if $r>1$, for all $\varphi \in \hat{W}_{q^{\prime}, \infty}^{1}(D)$ if $r=1$. When $1<q<3$, we have $u \in L_{q_{*}, r}(D)$ by (2.6), so that $u \rightarrow 0$ at infinity in this weak sense. When $(q, r)=(3,1)$, we know $u \rightarrow 0$ at infinity by Lemma 2.2 (iii).

In the whole space and in bounded domains, the notion of $(q, r)$-weak solution is defined similarly. For the exterior problem, in Section 5, we will prove that, for every $f \in \dot{W}_{q, r}^{-1}(D)$ with $(q, r)$ satisfying (1.11), there is a unique $(q, r)$-weak solution. However, for $f \in \dot{W}_{q, r}^{-1}(D)$ with $(q, r)$ satisfying (1.12), as in Shibata and Yamazaki [35], we have to enlarge the class of solutions. In this case, $(u, p)$ is called generalized $(q, r)$-weak solution of (2.9) if

1. $u \in \dot{W}_{q, r}^{1}(D)+\dot{W}_{3 / 2, \infty}^{1}(D), p \in L_{q, r}(D)+L_{3 / 2, \infty}(D)$;

2. $\operatorname{div} u=0$ in $L_{q, r}(D)+L_{3 / 2, \infty}(D)$;

3. $(\omega \wedge x) \cdot \nabla u-\omega \wedge u \in \dot{W}_{q, r}^{-1}(D)+\dot{W}_{3 / 2, \infty}^{-1}(D)$;

4. (2.10) holds for all $\varphi \in C_{0}^{\infty}(D)$.

In fact, one cannot expect faster decay of $(\nabla u, p)$ than $1 /|x|^{2}$, in general, even though the external force $f$ decays rapidly at infinity. On the other hand, when either $q>3$ or $q=3, r>1$, uniqueness of $(q, r)$-weak solution does not seem to hold.

Choose a cut-off function $\zeta \in C_{0}^{\infty}\left(\boldsymbol{R}^{3} ;[0,1]\right)$ satisfying $\zeta=1$ near the boundary $\partial D$, and set 


$$
b(x)=-\frac{1}{2} \operatorname{rot}\left(\zeta(x)|x|^{2} \omega\right)
$$

Obviously $\operatorname{div} b=0$ and $\left.b\right|_{\partial D}=\omega \wedge x$. We thus intend to find the solution to (1.2), (1.3) as $u=v+b$, so that $(v, p)$ should obey

$$
\begin{cases}-\Delta v-(\omega \wedge x) \cdot \nabla v+\omega \wedge v+\nabla p=f-\Phi(v, b) & \text { in } D, \\ \operatorname{div} v=0 & \text { in } D, \\ v=0 & \text { on } \partial D \\ v \rightarrow 0 & \text { at } \infty\end{cases}
$$

with

$$
\begin{aligned}
\Phi(v, b) & =(v+b) \cdot \nabla(v+b)+L b \\
& =\operatorname{div}[(v+b) \otimes(v+b)-\nabla b-(\omega \wedge x) \otimes b+b \otimes(\omega \wedge x)],
\end{aligned}
$$

where $w \otimes \tilde{w}=\left(w_{j} \tilde{w}_{k}\right)$; here, note that

$$
(\omega \wedge x) \cdot \nabla b=\operatorname{div}[(\omega \wedge x) \otimes b], \quad \omega \wedge b=\operatorname{div}[b \otimes(\omega \wedge x)] .
$$

Let $f \in \dot{W}_{3 / 2, \infty}^{-1}(D)$. Since $v \in \dot{W}_{3 / 2, \infty}^{1}(D)$ implies $\Phi(v, b) \in \dot{W}_{3 / 2, \infty}^{-1}(D)$, see Section 6 , one can define the weak solution $(v, p) \in \dot{W}_{3 / 2, \infty}^{1}(D) \times L_{3 / 2, \infty}(D)$ of $(2.12)$ as the $(3 / 2, \infty)$-weak solution of $(2.9)$ with $f$ replaced by $f-\Phi(v, b)$. In this sense $(u, p)=(v+b, p)$ is the weak solution of the original problem (1.2), (1.3).

\section{Linearized problem in the whole space}

Let us consider the whole space problem

$$
-\Delta u-(\omega \wedge x) \cdot \nabla u+\omega \wedge u+\nabla p=f, \quad \operatorname{div} \mathbf{u}=g \quad \text { in } \boldsymbol{R}^{3} .
$$

In the first half of this section we recall the $L_{q}$-theory developed in [28] and extend it to the case of Lorentz spaces. The second half is devoted to a proposition concerning the class of solutions when the support of $f$ is compact.

We begin with the $L_{q}$-estimate of weak solutions.

Proposition 3.1 ([28]). Let $1<q<\infty$ and suppose that

$$
f \in \dot{W}_{q}^{-1}\left(\boldsymbol{R}^{3}\right), \quad g \in L_{q}\left(\boldsymbol{R}^{3}\right), \quad(\omega \wedge x) g \in \dot{W}_{q}^{-1}\left(\boldsymbol{R}^{3}\right) .
$$

Then problem (3.1) possesses a q-weak solution $(u, p) \in \dot{W}_{q}^{1}\left(\boldsymbol{R}^{3}\right) \times L_{q}\left(\boldsymbol{R}^{3}\right)$ subject to the estimate

$$
\begin{aligned}
& \|\nabla u\|_{q, \boldsymbol{R}^{3}}+\|p\|_{q, \boldsymbol{R}^{3}}+\|(\omega \wedge x) \cdot \nabla u-\omega \wedge u\|_{\dot{W}_{q}^{-1}\left(\boldsymbol{R}^{3}\right)} \\
& \quad \leq C\left(\|f\|_{\dot{W}_{q}^{-1}\left(\boldsymbol{R}^{3}\right)}+\|g\|_{q, \boldsymbol{R}^{3}}+\|(\omega \wedge x) g\|_{\dot{W}_{q}^{-1}\left(\boldsymbol{R}^{3}\right)}\right),
\end{aligned}
$$


where $C=C(q)>0$ is independent of $|\omega|, f$ and $g$. The solution is unique in the class above up to a constant multiple of $\omega$ for $u$.

Although this result was proved in [28] only for the case $|\omega|=1$, a scaling argument implies that the constant $C>0$ in (3.2) is independent of $|\omega|$. By real interpolation we obtain the estimate of weak solutions in Lorentz spaces.

Proposition 3.2. Let $1<q<\infty, 1 \leq r \leq \infty$ and suppose that

$$
f \in \dot{W}_{q, r}^{-1}\left(\boldsymbol{R}^{3}\right), \quad g \in L_{q, r}\left(\boldsymbol{R}^{3}\right), \quad(\omega \wedge x) g \in \dot{W}_{q, r}^{-1}\left(\boldsymbol{R}^{3}\right) .
$$

Then problem (3.1) possesses a $(q, r)$-weak solution $(u, p) \in \dot{W}_{q, r}^{1}\left(\boldsymbol{R}^{3}\right) \times L_{q, r}\left(\boldsymbol{R}^{3}\right)$ subject to the estimate

$$
\begin{aligned}
& \|\nabla u\|_{q, r, \boldsymbol{R}^{3}}+\|p\|_{q, r, \boldsymbol{R}^{3}}+\|(\omega \wedge x) \cdot \nabla u-\omega \wedge u\|_{\dot{W}_{q, r}^{-1}\left(\boldsymbol{R}^{3}\right)} \\
& \leq C\left(\|f\|_{\dot{W}_{q, r}^{-1}\left(\boldsymbol{R}^{3}\right)}+\|g\|_{q, r, \boldsymbol{R}^{3}}+\|(\omega \wedge x) g\|_{\dot{W}_{q, r}^{-1}\left(\boldsymbol{R}^{3}\right)}\right),
\end{aligned}
$$

where $C=C(q, r)>0$ is independent of $|\omega|$. The solution is unique in the class above up to a constant multiple of $\omega$ for $u$.

Proof. We first obtain the pressure written formally by

$$
p=-\operatorname{div}(-\Delta)^{-1}[f+\nabla g+(\omega \wedge x) g]
$$

here, $(-\Delta)^{-1}$ can be considered as a bounded operator from $\dot{W}_{q}^{-1}\left(\boldsymbol{R}^{3}\right)$ to $\dot{W}_{q}^{1}\left(\boldsymbol{R}^{3}\right)$ ([22], [31]). By real interpolation it is bounded from $\dot{W}_{q, r}^{-1}\left(\boldsymbol{R}^{3}\right)$ to $\dot{W}_{q, r}^{1}\left(\boldsymbol{R}^{3}\right)$, see (2.4). Therefore,

$$
\|p\|_{q, r, \boldsymbol{R}^{3}} \leq C\|f+\nabla g+(\omega \wedge x) g\|_{\dot{W}_{q, r}^{-1}\left(\boldsymbol{R}^{3}\right)} .
$$

We next consider the equation $L u=f-\nabla p$. As shown in the proof of Proposition 3.1, the operator $L^{-1}$ is bounded from $\dot{W}_{q}^{-1}\left(\boldsymbol{R}^{3}\right)$ to $\dot{W}_{q}^{1}\left(\boldsymbol{R}^{3}\right)$; thus it is bounded from $\dot{W}_{q, r}^{-1}\left(\boldsymbol{R}^{3}\right)$ to $\dot{W}_{q, r}^{1}\left(\boldsymbol{R}^{3}\right)$ by real interpolation. Hence we obtain a solution $u \in \dot{W}_{q, r}^{1}\left(\boldsymbol{R}^{3}\right)$ with

$$
\|\nabla u\|_{q, r, \boldsymbol{R}^{3}} \leq C\|f-\nabla p\|_{\dot{W}_{q, r}^{-1}\left(\boldsymbol{R}^{3}\right)},
$$

where $C>0$ is independent of $|\omega|$, which combined with (3.4) implies (3.3). Finally, applying div to the first equation of (3.1), we get $L(\operatorname{div} u-g)=0$ yielding $\operatorname{div} u=g$, see below.

Let $(u, p) \in \dot{W}_{q, r}^{1}\left(\boldsymbol{R}^{3}\right) \times L_{q, r}\left(\boldsymbol{R}^{3}\right)$ satisfy $L u+\nabla p=0$, div $u=0$ in $\boldsymbol{R}^{3}$. We immediately obtain $p=0$, since $\Delta p=0$, so that $L u=0$. Since $\nabla u \in$ $L_{q, r}\left(\boldsymbol{R}^{3}\right) \subset \mathscr{S}^{\prime}$ and consequently also $u \in \mathscr{S}^{\prime}$, see [8, Proposition 1.2.1], we get supp $\hat{u} \subset\{0\}$ as shown in [14], [28]. Hence $u$ is a constant vector, which should be a constant multiple of $\omega$ because $\omega \wedge u=0$. This completes the proof. 
The final proposition on the linear whole space problem gives a heuristic argument why in spite of the negative result (1.1) the pointwise estimate (1.4) may hold. This proposition will be used only for the proof of uniqueness, see Proposition 5.1, but, differently from [35], not for the construction of solutions in exterior domains. It extends a similar result from [35] where $\omega=0$ and would be essential when employing a cut-off procedure as in [35].

Proposition 3.3. Let $1<q<\infty$ and suppose that

$$
f \in L_{q}\left(\boldsymbol{R}^{3}\right), \quad \operatorname{supp} f \subset B_{R}, \quad g=0,
$$

where $B_{R}=\left\{x \in \boldsymbol{R}^{3} ;|x|<R\right\}$. Then a representative $(u, p)$ of the strong solution to (3.1) obtained in [14] enjoys $(u, p) \in \dot{W}_{3 / 2, \infty}^{1}\left(\boldsymbol{R}^{3}\right) \times L_{3 / 2, \infty}\left(\boldsymbol{R}^{3}\right)$ and the estimates

$$
\begin{gathered}
\|\nabla u\|_{3 / 2, \infty, \boldsymbol{R}^{3}}+\|p\|_{3 / 2, \infty, \boldsymbol{R}^{3}} \leq C_{R}\|f\|_{q, \boldsymbol{R}^{3}} \\
\operatorname{ess} \sup _{|x| \geq 4 R}\left(|x||u(x)|+|x|^{2}(|\nabla u(x)|+|p(x)|)+|x|^{3}|\nabla p(x)|\right) \leq C_{R}\|f\|_{q, \boldsymbol{R}^{3}},
\end{gathered}
$$
with some $C_{R}=C_{R}(q)>0$ independent of $|\omega|$.

Proof. Since $\Delta p=\operatorname{div} f$, it follows from the Hausdorff-Young inequality that

$$
\|p\|_{3 / 2, \infty, \boldsymbol{R}^{3}} \leq C\|f\|_{1, \boldsymbol{R}^{3}}
$$

for $p(x)=-\frac{1}{4 \pi} \nabla \frac{1}{|\cdot|} * f$ and $\frac{1}{|\cdot|^{2}} \in L_{3 / 2, \infty}\left(\boldsymbol{R}^{3}\right)$. We also find

$$
\text { ess } \sup _{|x| \geq 2 R}\left(|x|^{2}|p(x)|+|x|^{3}|\nabla p(x)|\right) \leq C\|f\|_{1, \boldsymbol{R}^{3}},
$$

because of supp $f \subset B_{R}$.

By the relation $L_{q}\left(\boldsymbol{R}^{3}\right) \hookrightarrow \dot{W}_{3 q /(3-q)}^{-1}\left(\boldsymbol{R}^{3}\right)$, where we may assume $q<3$, Proposition 3.1 implies $\nabla u \in L_{3 q /(3-q)}\left(\boldsymbol{R}^{3}\right)$. Since $3 q /(3-q)>3 / 2$, we obtain

$$
\|\nabla u\|_{3 / 2, \infty, B_{4 R}} \leq C\|\nabla u\|_{3 q /(3-q), \boldsymbol{R}^{3}} \leq C\|f\|_{\dot{W}_{3 q /(3-q)}^{-1}\left(\boldsymbol{R}^{3}\right)} \leq C\|f\|_{q, \boldsymbol{R}^{3}},
$$

where $C=C_{R}>0$ is independent of $|\omega|$. For the proof of (3.5) and (3.6), therefore, it suffices to show

$$
\text { ess } \sup _{|x| \geq 4 R}|x|^{2}|\nabla u(x)| \leq C_{R}\|f\|_{1, \boldsymbol{R}^{3}},
$$

with some $C_{R}>0$ independent of $|\omega|$. To prove (3.10), we decompose $u=v+w$ where $v, w$ are defined by $L v=-\nabla p$ and $L w=f$ in $\boldsymbol{R}^{3}$, respectively. Using the fundamental solution matrix

$$
K(x, y)=\int_{0}^{\infty}(4 \pi t)^{-3 / 2} \exp \left(-\frac{|O(|\omega| t) x-y|^{2}}{4 t}\right) O(|\omega| t)^{T} d t
$$


of the operator $L$, see [14], [28], we first consider

$$
\begin{aligned}
\nabla v(x) & =-\int_{\boldsymbol{R}^{3}} \nabla_{x} K(x, y) \nabla_{y} p(y) d y \\
& =\left(-\int_{|y|<|x| / 2}-\int_{|y| \geq|x| / 2}\right)(\cdots) d y=: I_{0}(x)+I_{\infty}(x) .
\end{aligned}
$$

It is easily seen that

$$
\begin{aligned}
I_{\infty}(x)= & \int_{0}^{\infty}(4 \pi t)^{-3 / 2} \int_{|y| \geq|x| / 2} \exp \left(-\frac{|x-y|^{2}}{4 t}\right) \\
& \times\left[O(|\omega| t)^{T}(\nabla p)(O(|\omega| t) y)\right] \otimes \frac{x-y}{2 t} d y d t .
\end{aligned}
$$

Since $|y| \geq|x| / 2 \geq 2 R$ for $|x| \geq 4 R$, one can use (3.8) to obtain

$$
\left|I_{\infty}(x)\right| \leq C\|f\|_{1, \boldsymbol{R}^{3}} \int_{|y| \geq|x| / 2} \frac{|x-y|}{|y|^{3}} \int_{0}^{\infty} t^{-5 / 2} \exp \left(-\frac{|x-y|^{2}}{4 t}\right) d t d y .
$$

Recall

$$
\int_{0}^{\infty} t^{-\alpha} \exp \left(-\frac{|x|^{2}}{c t}\right) d t=\frac{c^{\alpha-1} \Gamma(\alpha-1)}{|x|^{2(\alpha-1)}}, \quad x \neq 0, \alpha>1, c>0,
$$

where $\Gamma(\cdot)$ is the Gamma function, so that

$$
\left|I_{\infty}(x)\right| \leq C\|f\|_{1, \boldsymbol{R}^{3}} \int_{|y| \geq|x| / 2} \frac{1}{|y|^{3}|x-y|^{2}} d y .
$$

Using the change of variable $y^{\prime}=y /|x|$, it is easily seen that the integral above is bounded by $c /|x|^{2}$. We thus obtain

$$
\left|I_{\infty}(x)\right| \leq \frac{C\|f\|_{1, \boldsymbol{R}^{3}}}{|x|^{2}}, \quad|x| \geq 4 R
$$

Integration by parts yields

$$
I_{0}(x)=I_{01}(x)+I_{02}(x)
$$

with

$$
\begin{aligned}
I_{01}(x)= & \int_{0}^{\infty}(4 \pi t)^{-3 / 2} \int_{|y|=|x| / 2} \exp \left(-\frac{|x-y|^{2}}{4 t}\right) \\
& \times \frac{\left(O(|\omega| t)^{T} \frac{y}{|y|}\right) \otimes(x-y)}{2 t} p(O(|\omega| t) y) d \sigma_{y} d t
\end{aligned}
$$


and

$$
\begin{aligned}
I_{02}(x)= & \int_{0}^{\infty}(4 \pi t)^{-3 / 2} \int_{|y|<|x| / 2} \exp \left(-\frac{|x-y|^{2}}{4 t}\right) \\
& \times\left(\frac{(x-y) \otimes(x-y)}{-4 t^{2}}+\frac{\boldsymbol{I}}{2 t}\right) p(O(|\omega| t) y) d y d t,
\end{aligned}
$$

where $\boldsymbol{I}=\left(\delta_{j k}\right)$. For $|y|=|x| / 2 \geq 2 R$, we have $|p(O(t) y)| \leq C|x|^{-2}\|f\|_{1, \boldsymbol{R}^{3}}$ by (3.8). This together with (3.11) implies

$$
\left|I_{01}(x)\right| \leq \frac{C\|f\|_{1, \boldsymbol{R}^{3}}}{|x|^{2}} \int_{|y|=|x| / 2} \frac{d \sigma_{y}}{|x-y|^{2}} \leq \frac{C\|f\|_{1, \boldsymbol{R}^{3}}}{|x|^{2}}, \quad|x| \geq 4 R .
$$

Since $|x| / 2<|x-y|<3|x| / 2$ for $|y|<|x| / 2$, it follows from (3.11) and (3.8) that

$$
\begin{aligned}
\left|I_{02}(x)\right| & \leq C \int_{0}^{\infty}\left(t^{-7 / 2}|x|^{2}+t^{-5 / 2}\right) \exp \left(-\frac{|x|^{2}}{16 t}\right) d t \int_{|y|<|x| / 2}|p(y)| d y \\
& \leq \frac{C}{|x|^{3}}\left(\|p\|_{1, B_{2 R}}+C\|f\|_{1, \boldsymbol{R}^{3}} \int_{B_{|x| / 2}|y|^{2}} \frac{d y}{\mid x .}\right.
\end{aligned}
$$

By (3.7) we find $\|p\|_{1, B_{2 R}} \leq C_{R}\|p\|_{3 / 2, \infty, \boldsymbol{R}^{3}} \leq C_{R}\|f\|_{1, \boldsymbol{R}^{3}}$. As a consequence,

$$
\left|I_{02}(x)\right| \leq \frac{C\|f\|_{1, \boldsymbol{R}^{3}}}{|x|^{3}}\left(C_{R}+|x|\right), \quad|x| \geq 4 R .
$$

We collect (3.12), (3.13) and (3.14) to get

$$
\text { ess } \sup _{|x| \geq 4 R}|x|^{2}|\nabla v(x)| \leq C_{R}\|f\|_{1, \boldsymbol{R}^{3}},
$$

with some $C_{R}>0$ independent of $|\omega|$.

Finally, we see from supp $f \subset B_{R}$ that

$$
|\nabla w(x)| \leq C \int_{0}^{\infty} t^{-5 / 2} \int_{B_{R}} \exp \left(-\frac{|x-y|^{2}}{4 t}\right)|x-y||f(O(|\omega| t) y)| d y d t .
$$

Since $|y|<R<|x| / 2$, we have $|x| / 2<|x-y|<3|x| / 2$, so that

$$
|\nabla w(x)| \leq C|x| \int_{0}^{\infty} t^{-5 / 2} \exp \left(-\frac{|x|^{2}}{16 t}\right) d t \int_{B_{R}}|f(y)| d y,
$$

yielding with the help of (3.11) the estimate

$$
\text { ess } \sup _{|x| \geq 4 R}|x|^{2}|\nabla w(x)| \leq C\|f\|_{1, \boldsymbol{R}^{3}} .
$$


This inequality combined with (3.15) implies (3.10). By analogy, we get the estimate $|x||u(x)| \leq C\|f\|_{1, \boldsymbol{R}^{3}}$. Now we have completed the proof.

\section{Linearized problem in bounded domains}

Given a bounded domain $\Omega \subset \boldsymbol{R}^{3}$ with smooth boundary $\partial \Omega$ we consider weak solutions to the boundary value problem

$$
\begin{cases}-\Delta u-(\omega \wedge x) \cdot \nabla u+\omega \wedge u+\nabla p=f & \text { in } \Omega, \\ \operatorname{div} u=g & \text { in } \Omega, \\ u=0 & \text { on } \partial \Omega .\end{cases}
$$

For the usual Stokes problem, the case $\omega=0$, the following results are known, see Cattabriga [7], Solonnikov [37], Kozono and Sohr [30] and Kozono and Yamazaki [32]. The second part (ii) follows from (i) by real interpolation.

Lemma 4.1. Let $\Omega \subset \boldsymbol{R}^{3}$ be a bounded domain with smooth boundary, let $1<q<\infty$ and consider problem (4.1) with $\omega=0$.

(i) ([7], [37], [30]) Suppose that

$$
f \in \dot{W}_{q}^{-1}(\Omega), \quad g \in L_{q}(\Omega), \quad \int_{\Omega} g(x) d x=0 .
$$

Then there exists a unique q-weak solution $(u, p) \in \dot{W}_{q}^{1}(\Omega) \times L_{q}(\Omega)$ (up to an additive constant for $p$ ) subject to the estimate

$$
\|\nabla u\|_{q, \Omega}+\|u\|_{q, \Omega}+\|p-\bar{p}\|_{q, \Omega} \leq C\left(\|f\|_{\dot{W}_{q}^{-1}(\Omega)}+\|g\|_{q, \Omega}\right),
$$

where $\bar{p}=\frac{1}{|\Omega|} \int_{\Omega} p(x) d x$.

(ii) ([32, Lemma 2.7]) Let $1 \leq r \leq \infty$. Suppose that

$$
f \in \dot{W}_{q, r}^{-1}(\Omega), \quad g \in L_{q, r}(\Omega), \quad \int_{\Omega} g(x) d x=0 .
$$

Then there exists a unique $(q, r)$-weak solution $(u, p) \in \dot{W}_{q, r}^{1}(\Omega) \times L_{q, r}(\Omega)$ (up to an additive constant for $p$ ) subject to the estimate

$$
\|\nabla u\|_{q, r, \Omega}+\|u\|_{q, r, \Omega}+\|p-\bar{p}\|_{q, r, \Omega} \leq C\left(\|f\|_{\dot{W}_{q, r}^{-1}(\Omega)}+\|g\|_{q, r, \Omega}\right) .
$$

In bounded domains, the operator $L$ can be treated as a perturbation to the Laplace operator. For the argument in the next section it suffices to consider the case $\operatorname{div} u=g=0$.

Proposition 4.1. Let $\Omega \subset \boldsymbol{R}^{3}$ be a bounded domain with smooth boundary, and let $1<q<\infty, 1 \leq r \leq \infty$. Suppose that $f \in \dot{W}_{q, r}^{-1}(\Omega)$ and $g=0$. Then 
problem (4.1) possesses a unique $(q, r)$-weak solution $(u, p) \in \dot{W}_{q, r}^{1}(\Omega) \times L_{q, r}(\Omega)$ (up to an additive constant for $p$ ) subject to the estimate

$$
\|\nabla u\|_{q, r, \Omega}+\|u\|_{q, r, \Omega}+\|p-\bar{p}\|_{q, r, \Omega} \leq C\|f\|_{\dot{W}_{q, r}^{-1}(\Omega)},
$$

with some $C=C(q, r, M)>0$ uniformly in $|\omega| \in[0, M], M>0$, where $\bar{p}$ is as in Lemma 4.1.

Proof. Set

$$
D u=\left(e_{3} \wedge x\right) \cdot \nabla u-e_{3} \wedge u=\operatorname{div}\left[\left(e_{3} \wedge x\right) \otimes u-u \otimes\left(e_{3} \wedge x\right)\right] .
$$

Then (4.1) can be rewritten as

$$
-\Delta u+\nabla p=f+|\omega| D u, \quad \operatorname{div} u=0 .
$$

Let $T: \dot{W}_{q, r}^{-1}(\Omega) \rightarrow \dot{W}_{q, r}^{1}(\Omega), f \mapsto u$, be the solution operator defined by Lemma 4.1 (ii) with $g=0$. Given $f \in \dot{W}_{q, r}^{-1}(\Omega)$, we intend to solve

$$
(1-|\omega| T D) u=T f
$$

in $\dot{W}_{q, r}^{1}(\Omega)$. Lemma 4.1 together with $(2.5)$ yields

$$
\|\nabla T D v\|_{q, r, \Omega} \leq C\|D v\|_{\dot{W}_{q, r}^{-1}(\Omega)} \leq C\|v\|_{q, r, \Omega} \leq c\|\nabla v\|_{q, r, \Omega}
$$

for some $c=c(q, r, \Omega)>0$ independent of $v \in \dot{W}_{q, r}^{1}(\Omega)$. Therefore, the operator $T D: L_{q, r}(\Omega) \rightarrow \dot{W}_{q, r}^{1}(\Omega)$ is bounded and, due to Lemma 2.1, even compact as an operator from $\dot{W}_{q, r}^{1}(\Omega)$ into itself. Moreover, we use the injectivity of the operator $1-|\omega| T D: \dot{W}_{q, r}^{1}(\Omega) \rightarrow \dot{W}_{q, r}^{1}(\Omega)$. In fact, the equation $(1-|\omega| T D) u$ $=0$ is equivalent to the Stokes system $-\Delta u+\nabla p=|\omega| D u$, div $u=0$ where $|\omega| D u \in L_{q, r}(\Omega)$. Then a bootstrapping argument yields $u \in \dot{W}_{2}^{1}(\Omega)$, and testing the equation with $u$ itself shows that $u=0$.

Now Fredholm theory proves that the operator $1-|\omega| T D$ has a bounded inverse. Hence the unique solution $u$ to (4.1) satisfies the estimate

$$
\|\nabla u\|_{q, r, \Omega} \leq C_{\omega}\|f\|_{\dot{W}_{q, r}^{-1}(\Omega)} .
$$

We also obtain an associated pressure $p$ from Lemma 4.1 satisfying

$$
\|p-\bar{p}\|_{q, r, \Omega} \leq C_{\omega}\|f+|\omega| D u\|_{\dot{W}_{q, r}^{-1}(\Omega)} \leq C_{\omega}\|f\|_{\dot{W}_{q, r}^{-1}(\Omega)} .
$$

So far the constant $C_{\omega}$ will depend somehow on $\omega$. However, an argument by contradiction as in the proof of Theorem 5.1 will easily prove that $C_{\omega}=C(M)$ uniformly in $|\omega| \in[0, M], M>0$, where (4.3) is used instead of (5.19). This completes the proof.

Finally we mention a well-known result on the divergence problem. 
Lemma 4.2 ([2], [6], [17]). Let $\Omega \subset \boldsymbol{R}^{n}$ be a bounded domain with Lipschitz boundary. Let $1<q<\infty$ and $k \geq 0$ integer. Then there is a linear operator $B: C_{0}^{\infty}(\Omega) \rightarrow C_{0}^{\infty}(\Omega)^{n}$ such that

$$
\left\|\nabla^{k+1} B f\right\|_{q, \Omega} \leq C\left\|\nabla^{k} f\right\|_{q, \Omega}
$$

with some $C=C(\Omega, q, k)>0$ and that

$$
\operatorname{div}(B f)=f \quad \text { if } \int_{\Omega} f(x) d x=0 .
$$

By continuity, $B$ is extended uniquely to a bounded operator from $\dot{W}_{q}^{k}(\Omega)$ to $\dot{W}_{q}^{k+1}(\Omega)^{n}$, where $\dot{W}_{q}^{k}(\Omega)$ is the completion of $C_{0}^{\infty}(\Omega)$ with respect to the norm $\left\|\nabla^{k}(\cdot)\right\|_{q, \Omega}$. Furthermore, by real interpolation, it is extended uniquely to a bounded operator from $\dot{W}_{q, r}^{k}(\Omega)$ to $\dot{W}_{q, r}^{k+1}(\Omega)^{n}$, where $1 \leq r \leq \infty$ and

$$
\dot{W}_{q, r}^{k}(\Omega)=\left(\dot{W}_{q_{0}}^{k}(\Omega), \dot{W}_{q_{1}}^{k}(\Omega)\right)_{\theta, r}
$$

with $q_{0}, q_{1}$ and $\theta$ satisfying (2.3).

\section{Linearized problem in exterior domains}

This section establishes Theorem 5.1 and Theorem 5.2 below on existence, uniqueness and estimate for solutions of the exterior boundary value problem

$$
L u+\nabla p=f, \quad \operatorname{div} u=0 \quad \text { in } D ;\left.\quad u\right|_{\partial D}=0 .
$$

The first theorem is concerned with $(q, r)$-weak solutions.

Theorem 5.1. Let $(q, r)$ satisfy one of the conditions in (1.11) and let $f \in \dot{W}_{q, r}^{-1}(D)$. Then problem (5.1) possesses a unique $(q, r)$-weak solution

$$
(u, p) \in \dot{W}_{q, r}^{1}(D) \times L_{q, r}(D)
$$

subject to the estimate

$$
\|(\nabla u, p)\|_{q, r}+\|(\omega \wedge x) \cdot \nabla u-\omega \wedge u\|_{\dot{W}_{q, r}^{-1}(D)} \leq C\|f\|_{\dot{W}_{q, r}^{-1}(D)},
$$

with some $C=C(q, r, M)>0$ uniformly in $|\omega| \in[0, M], M>0$.

The next theorem studies the case of generalized $(q, r)$-weak solutions when $(q, r)$ satisfies (1.12).

Theorem 5.2. Let $(q, r)$ satisfy one of the conditions in (1.12) and let $f \in \dot{W}_{q, r}^{-1}(D)$. Then problem (5.1) possesses a unique generalized $(q, r)$-weak solution

$$
\left\{\begin{array}{l}
u=v+\tilde{v}, \quad p=\pi+\tilde{\pi} \\
(v, \pi) \in \dot{W}_{q, r}^{1}(D) \times L_{q, r}(D), \quad(\tilde{v}, \tilde{\pi}) \in \dot{W}_{3 / 2, \infty}^{1}(D) \times L_{3 / 2, \infty}(D)
\end{array}\right.
$$


subject to the estimate

$$
\|(\nabla v, \pi)\|_{q, r}+\|(\nabla \tilde{v}, \tilde{\pi})\|_{3 / 2, \infty} \leq C\|f\|_{\dot{W}_{q, r}^{-1}(D)},
$$

with some $C=C(q, r, M)>0$ uniformly in $|\omega| \in[0, M], M>0$.

In both theorems the smallness of $|\omega|$ is unnecessary, although the constant $C$ may be growing when $|\omega|$ is increasing. In Theorem 5.2, we have $(\omega \wedge x) \cdot \nabla \tilde{v}-\omega \wedge \tilde{v} \in \dot{W}_{3 / 2, \infty}^{-1}(D)$, while $(\omega \wedge x) \cdot \nabla v-\omega \wedge v$ does not necessarily belong to $\dot{W}_{q, r}^{-1}(D)$, but to $\dot{W}_{q, r}^{-1}(D)+\dot{W}_{3 / 2, \infty}^{-1}(D)$. This will be made clear in the proof.

We introduce a few cut-off functions used for the proof of both uniqueness and existence. Fix $\rho>0$ so large that $\boldsymbol{R}^{3} \backslash D \subset B_{\rho-5}$, and choose the functions $\phi_{j} \in C^{\infty}\left(\boldsymbol{R}^{3} ;[0,1]\right), j=0,1,2$, satisfying

$$
\phi_{1}(x)=\left\{\begin{array}{ll}
0, & |x| \leq \rho-5, \\
1, & |x| \geq \rho-4,
\end{array} \quad \phi_{j}(x)=\left\{\begin{array}{ll}
1, & |x| \leq \rho-3+j, \\
0, & |x| \geq \rho-2+j,
\end{array} \quad j=0,2 .\right.\right.
$$

We set

$$
D_{\rho}=D \cap B_{\rho}, \quad A_{\rho}=\left\{x \in \boldsymbol{R}^{3} ; \rho-4<|x|<\rho-1\right\} .
$$

We begin with the question of uniqueness of $(q, r)$-weak solutions.

Proposition 5.1. Assume that either $1<q<3,1 \leq r \leq \infty$ or $(q, r)=(3,1)$. Let $f=0$. Then the only $(q, r)$-weak solution $(u, p) \in \dot{W}_{q, r}^{1}(D) \times L_{q, r}(D)$ for (5.1) is the trivial one, that is, $(u, p)=(0,0)$.

Proof. We use the cut-off function $\phi_{0}$ given by (5.5) and the operator $B$ defined by Lemma 4.2 in the bounded domain $A_{\rho}$, see (5.6). Given a $(q, r)$ weak solution $(u, p)$ of $(5.1)$ with $f=0$, we set

$$
v=\left(1-\phi_{0}\right) u+B\left[u \cdot \nabla \phi_{0}\right], \quad \pi=\left(1-\phi_{0}\right) p .
$$

Since $\int_{A_{\rho}} u \cdot \nabla \phi_{0} d x=0$, we have $\operatorname{div} v=0$. Thus $(v, \pi)$ is a distribution solution to

$$
L v+\nabla \pi=F, \quad \operatorname{div} v=0 \quad \text { in } \boldsymbol{R}^{3},
$$

where

$$
F=2 \nabla \phi_{0} \cdot \nabla u+\left[\Delta \phi_{0}+(\omega \wedge x) \cdot \nabla \phi_{0}\right] u-\left(\nabla \phi_{0}\right) p+L B\left[u \cdot \nabla \phi_{0}\right] .
$$

In view of Lemma 2.2 (ii) or (iii) and Lemma 4.2, we have $F \in L_{\tilde{q}}\left(\boldsymbol{R}^{3}\right)$ for all $\tilde{q} \in(1, q)$ with $\operatorname{supp} F \subset A_{\rho}$; hence $(v, \pi)$ is a strong solution to (5.7). It follows from Lemma 2.2 (ii) or (iii) that $u$ is small at infinity and, therefore, so is $v$. Consequently, $v$ is represented in terms of the fundamental solution matrix, see the proof of Proposition 3.3. We thus obtain from Proposition 3.3 that $(v, \pi)$ 
satisfies (3.6) with the right-hand side $C_{\rho}\|F\|_{\tilde{q}, \boldsymbol{R}^{3}}$. Since $(u, p)$ coincides with $(v, \pi)$ for $|x| \geq \rho-1$, we find

$$
|u(x)|=O\left(|x|^{-1}\right), \quad|\nabla u(x)|+|p(x)|=O\left(|x|^{-2}\right)
$$

as $|x| \rightarrow \infty$. Note that the key idea of this proof is the smallness of $u$ at infinity in a weak sense implying the decay properties (5.8).

Now, let $\psi \in C^{\infty}([0, \infty) ;[0,1])$ satisfy $\psi(t)=1$ for $0 \leq t \leq 1$ and $\psi(t)=0$ for $t \geq 2$, and set $\psi_{R}(x)=\psi(|x| / R)$ for $R>\rho$ and $x \in \boldsymbol{R}^{3}$. We multiply (5.1) with $f=0$ by $\psi_{R} u$ which is allowed since a bootstrapping argument using local regularity results for the usual Stokes problem in exterior domains yields $(u, p) \in W_{2}^{2}\left(D_{2 R}\right) \times W_{2}^{1}\left(D_{2 R}\right)$. Note that $(\omega \wedge u) \cdot\left(\psi_{R} u\right)=0$ and that

$$
\int_{D_{2 R}}[(\omega \wedge x) \cdot \nabla u] \cdot\left(\psi_{R} u\right) d x=\frac{-1}{2} \int_{D_{2 R}}|u|^{2}(\omega \wedge x) \cdot \nabla \psi_{R} d x=0
$$

since

$$
(\omega \wedge x) \cdot \nabla \psi_{R}(x)=\psi^{\prime}\left(\frac{|x|}{R}\right) \frac{(\omega \wedge x) \cdot x}{R|x|}=0 .
$$

Therefore, as in the usual Stokes problem, we find

$$
\int_{D}|\nabla u|^{2} \psi_{R} d x+\int_{R<|x|<2 R}\left(\nabla u \cdot \nabla \psi_{R}\right) \cdot u d x-\int_{R<|x|<2 R}\left(u \cdot \nabla \psi_{R}\right) p d x=0 .
$$

By virtue of (5.8) together with $\left|\nabla \psi_{R}(x)\right| \leq C / R$ we see that both the second and third terms are estimated from above by $C / R$. Letting $R \rightarrow \infty$ yields $\int_{D}|\nabla u|^{2} d x=0$, so that $u=0$ by the boundary condition. We have also $\nabla p=0$, which together with (5.8) implies $p=0$.

For $(q, r)$ satisfying (1.12), one can also show the uniqueness of weak solutions in the class (5.3) given by Theorem 5.2 along the same line as in the proof of Proposition 5.1 because (5.3) implies (5.8).

Proposition 5.2. Let $(q, r)$ satisfy one of the conditions in (1.12) and $f=0$. Then the only generalized (q,r)-weak solution ( $u, p)$ of class (5.3) for (5.1) is the trivial one, that is, $(u, p)=(0,0)$.

We next consider the existence part of Theorem 5.1. Since the result for the case $3 / 2<q<3$ is obtained by real interpolation of the existence theorem in [28], our task is to construct solutions for the cases $(q, r)=(3 / 2, \infty)$ and $(q, r)=(3,1)$.

We first discuss the former case which is actually needed to solve the Navier-Stokes problem. For given $f \in \dot{W}_{3 / 2, \infty}^{-1}(D)$ we will construct the solution of (5.1) by means of a cut-off technique, using the solutions in the whole 
space (Section 3) and in a bounded domain (Section 4) together with the cut-off functions (5.5).

Consider (3.1) with $f$ replaced by $\phi_{1} f$ and $g=0$ in the whole space $\boldsymbol{R}^{3}$. We see that $\phi_{1} f \in \dot{W}_{3 / 2, \infty}^{-1}\left(\boldsymbol{R}^{3}\right)$ with

$$
\left\|\phi_{1} f\right\|_{\dot{W}_{3 / 2, \infty}^{-1}\left(\boldsymbol{R}^{3}\right)} \leq C\|f\|_{\dot{W}_{3 / 2, \infty}^{-1}(D)} .
$$

This is proved by using $\dot{W}_{3,1}^{1}\left(\boldsymbol{R}^{3}\right) \hookrightarrow L_{\infty}\left(\boldsymbol{R}^{3}\right)$, see $(2.8)$; in fact, for all $\psi \in C_{0}^{\infty}\left(\boldsymbol{R}^{3}\right)$, we find

$$
\begin{aligned}
\left|\left\langle\phi_{1} f, \psi\right\rangle\right| & \leq\|f\|_{\dot{W}_{3 / 2, \infty}^{-1}(D)}\left\|\nabla\left(\phi_{1} \psi\right)\right\|_{3,1} \\
& \leq C\|f\|_{\dot{W}_{3 / 2, \infty}^{-1}(D)}\left(\|\psi\|_{\infty, A_{\rho}}+\|\nabla \psi\|_{3,1}\right) \\
& \leq C\|f\|_{\dot{W}_{3 / 2, \infty}^{-1}(D)}\|\nabla \psi\|_{3,1, \boldsymbol{R}^{3}} .
\end{aligned}
$$

Let $\left(u_{\infty}, p_{\infty}\right)$ be the solenoidal solution obtained in Proposition 3.2 for the external force $\phi_{1} f$, and let

$$
\left(S_{\infty}, \Pi_{\infty}\right): \dot{W}_{3 / 2, \infty}^{-1}(D) \ni f \mapsto\left(u_{\infty}, p_{\infty}\right) \in \dot{W}_{3 / 2, \infty}^{1}\left(\boldsymbol{R}^{3}\right) \times L_{3 / 2, \infty}\left(\boldsymbol{R}^{3}\right)
$$

denote the solution operator. Here, $u_{\infty}$ is uniquely chosen in such a way that $u_{\infty} \in L_{3, \infty}\left(\boldsymbol{R}^{3}\right)$.

We also consider (4.1) with $f$ replaced by $\phi_{2} f$ and $g=0$ in the bounded domain $\Omega=D_{\rho}$, see (5.6). We easily see that $\phi_{2} f \in \dot{W}_{3 / 2, \infty}^{-1}\left(D_{\rho}\right)$ with

$$
\left\|\phi_{2} f\right\|_{\dot{W}_{3 / 2, \infty}^{-1}\left(D_{\rho}\right)} \leq C\|f\|_{\dot{W}_{3 / 2, \infty}^{-1}(D)} .
$$

Let $\left(u_{0}, p_{0}\right)$ be the solution obtained in Proposition 4.1 for the external force $\phi_{2} f$, and let

$$
\left(S_{0}, \Pi_{0}\right): \dot{W}_{3 / 2, \infty}^{-1}(D) \ni f \mapsto\left(u_{0}, p_{0}\right) \in \dot{W}_{3 / 2, \infty}^{1}\left(D_{\rho}\right) \times L_{3 / 2, \infty}\left(D_{\rho}\right)
$$

denote the solution operator. Here, $p_{0}$ is chosen such that $\int_{D_{\rho}} p_{0}(x) d x=0$.

As a parametrix (an approximation of the solution) for the exterior problem (5.1), we take

$$
\left\{\begin{array}{l}
S f=\left(1-\phi_{0}\right) S_{\infty} f+\phi_{0} S_{0} f+B\left[\left(S_{\infty} f-S_{0} f\right) \cdot \nabla \phi_{0}\right], \\
\Pi f=\left(1-\phi_{0}\right) \Pi_{\infty} f+\phi_{0} \Pi_{0} f
\end{array}\right.
$$

where $B$ is the operator defined by Lemma 4.2 in the bounded domain $A_{\rho}$. Note that

$$
\int_{A_{\rho}}\left(S_{\infty} f-S_{0} f\right) \cdot \nabla \phi_{0} d x=0,
$$

which implies $\operatorname{div}(S f)=0$. Concerning the class of $(S f, \Pi f)$, we have 
Proposition 5.3. Let $f \in \dot{W}_{3 / 2, \infty}^{-1}(D)$ be given. Then

$$
(S f, \Pi f) \in \dot{W}_{3 / 2, \infty}^{1}(D) \times L_{3 / 2, \infty}(D)
$$

and

$$
\|\nabla S f\|_{3 / 2, \infty}+\|\Pi f\|_{3 / 2, \infty} \leq C\|f\|_{\dot{W}_{3 / 2, \infty}^{-1}(D)}
$$

for some $C=C(M)>0$ uniformly in $|\omega| \in[0, M], M>0$.

Proof. By Lemma 4.2 we obtain

$$
\begin{aligned}
\|\nabla S f\|_{3 / 2, \infty} & \leq\left\|\nabla u_{\infty}\right\|_{3 / 2, \infty, \boldsymbol{R}^{3}}+\left\|\nabla u_{0}\right\|_{3 / 2, \infty, D_{\rho}}+C\left\|u_{\infty}-u_{0}\right\|_{3 / 2, \infty, A_{\rho}}, \\
\|\Pi f\|_{3 / 2, \infty} & \leq\left\|p_{\infty}\right\|_{3 / 2, \infty, \boldsymbol{R}^{3}}+\left\|p_{0}\right\|_{3 / 2, \infty, D_{\rho}},
\end{aligned}
$$

where $\left(u_{\infty}, p_{\infty}\right):=\left(S_{\infty} f, \Pi_{\infty} f\right)$ and $\left(u_{0}, p_{0}\right):=\left(S_{0} f, \Pi_{0} f\right)$. The Sobolev inequality (2.7) in $\boldsymbol{R}^{3}$ and the Poincare inequality (2.5) in $D_{\rho}$ lead us to the estimates

$$
\begin{gathered}
\left\|u_{\infty}\right\|_{3 / 2, \infty, A_{\rho}} \leq C\left\|u_{\infty}\right\|_{3, \infty, \boldsymbol{R}^{3}} \leq C\left\|\nabla u_{\infty}\right\|_{3 / 2, \infty, \boldsymbol{R}^{3}}, \\
\left\|u_{0}\right\|_{3 / 2, \infty, A_{\rho}} \leq\left\|u_{0}\right\|_{3 / 2, \infty, D_{\rho}} \leq C\left\|\nabla u_{0}\right\|_{3 / 2, \infty, D_{\rho}} .
\end{gathered}
$$

Thus Propositions 3.2 and 4.1 imply

$$
\|\nabla S f\|_{3 / 2, \infty}+\|\Pi f\|_{3 / 2, \infty} \leq C\left(\left\|\phi_{1} f\right\|_{\dot{W}_{3 / 2, \infty}^{-1}\left(\boldsymbol{R}^{3}\right)}+\left\|\phi_{2} f\right\|_{\dot{W}_{3 / 2, \infty}^{-1}\left(D_{\rho}\right)}\right),
$$

which together with (5.9) and (5.10) gives the estimate (5.12). We also have $S f+a \in L_{3, \infty}(D)$ for some $a \in \boldsymbol{R}^{3}$, see Lemma 2.2. But since $S f=S_{\infty} f$ for large $|x|$ and $S_{\infty} f \in \dot{W}_{3 / 2, \infty}^{1}\left(\boldsymbol{R}^{3}\right) \hookrightarrow L_{3, \infty}\left(\boldsymbol{R}^{3}\right)$, we find $a=0$. We have proved $S f \in \dot{W}_{3 / 2, \infty}^{1}(D)$.

We see that $(v, \pi)=(S f, \Pi f)$ is a distribution solution to

$$
L v+\nabla \pi=f+R f, \quad \operatorname{div} v=0 \quad \text { in } D ;\left.\quad v\right|_{\partial D}=0,
$$

where the remainder term $R f$ is defined as

$$
\begin{aligned}
R f= & -2 \nabla \phi_{0} \cdot \nabla\left(S_{\infty} f-S_{0} f\right)-\left[\Delta \phi_{0}+(\omega \wedge x) \cdot \nabla \phi_{0}\right]\left(S_{\infty} f-S_{0} f\right) \\
& -L B\left[\left(S_{\infty} f-S_{0} f\right) \cdot \nabla \phi_{0}\right]+\left(\nabla \phi_{0}\right)\left(\Pi_{\infty} f-\Pi_{0} f\right) .
\end{aligned}
$$

Lemma 5.1. Suppose that $f \in \dot{W}_{3 / 2, \infty}^{-1}(D)$. Then we have $R f \in$ $L_{3 / 2, \infty}\left(A_{\rho}\right) \cap \dot{W}_{3 / 2, \infty}^{-1}(D)$ and

$$
\|R f\|_{3 / 2, \infty, A_{\rho}}+\|R f\|_{\dot{W}_{3 / 2, \infty}^{-1}(D)} \leq C\|f\|_{\dot{W}_{3 / 2, \infty}^{-1}(D)} .
$$

Proof. We start with the estimate of $R f$ in $\dot{W}_{3 / 2, \infty}^{-1}(D)$. Let $\psi \in C_{0}^{\infty}(D)$. By (2.8) we have, for any $r \in(1, \infty)$, 


$$
\|\psi\|_{r, 1, A_{\rho}} \leq C\|\psi\|_{\infty, A_{\rho}} \leq C\|\psi\|_{\infty, D} \leq C\|\nabla \psi\|_{3,1, D},
$$

from which together with Lemma 4.2 it follows that

$$
\begin{aligned}
& \left|\left\langle 2 \nabla \phi_{0} \cdot \nabla u_{\infty}+\left[\Delta \phi_{0}+(\omega \wedge x) \cdot \nabla \phi_{0}\right] u_{\infty}-\left(\nabla \phi_{0}\right) p_{\infty}, \psi\right\rangle\right| \\
& \quad \leq C\left(\left\|\nabla u_{\infty}\right\|_{3 / 2, \infty, \boldsymbol{R}^{3}}+\left\|u_{\infty}\right\|_{3, \infty, \boldsymbol{R}^{3}}+\left\|p_{\infty}\right\|_{3 / 2, \infty, \boldsymbol{R}^{3}}\right)\|\psi\|_{\infty, A_{\rho}} \\
& \quad \leq C\left(\left\|\nabla u_{\infty}\right\|_{3 / 2, \infty, \boldsymbol{R}^{3}}+\left\|p_{\infty}\right\|_{3 / 2, \infty, \boldsymbol{R}^{3}}\right)\|\nabla \psi\|_{3,1, D},
\end{aligned}
$$

and that

$$
\begin{aligned}
\left|\left\langle L B\left[u_{\infty} \cdot \nabla \phi_{0}\right], \psi\right\rangle\right| & \leq C\left\|L B\left[u_{\infty} \cdot \nabla \phi_{0}\right]\right\|_{3 / 2, \infty, A_{\rho}}\|\psi\|_{3,1, A_{\rho}} \\
& \leq C\left(\left\|\nabla u_{\infty}\right\|_{3 / 2, \infty, A_{\rho}}+\left\|u_{\infty}\right\|_{3 / 2, \infty, A_{\rho}}\right)\|\psi\|_{\infty, A_{\rho}} \\
& \leq C\left(\left\|\nabla u_{\infty}\right\|_{3 / 2, \infty, \boldsymbol{R}^{3}}+\left\|u_{\infty}\right\|_{3, \infty, \boldsymbol{R}^{3}}\right)\|\nabla \psi\|_{3,1, D} \\
& \leq C\left\|\nabla u_{\infty}\right\|_{3 / 2, \infty, \boldsymbol{R}^{3}}\|\nabla \psi\|_{3,1, D},
\end{aligned}
$$

where $\left(u_{\infty}, p_{\infty}\right)=\left(S_{\infty} f, \Pi_{\infty} f\right)$. The terms including $\left(S_{0} f, \Pi_{0} f\right)$ can easily be estimated. As a result, we obtain $R f \in \dot{W}_{3 / 2, \infty}^{-1}(D)$ and, by Propositions 3.2 and 4.1

$$
\begin{aligned}
& \|R f\|_{\dot{W}_{3 / 2, \infty}^{-1}(D)} \\
& \quad \leq C\left(\left\|\nabla u_{\infty}\right\|_{3 / 2, \infty, \boldsymbol{R}^{3}}+\left\|p_{\infty}\right\|_{3 / 2, \infty, \boldsymbol{R}^{3}}+\left\|\nabla u_{0}\right\|_{3 / 2, \infty, D_{\rho}}+\left\|p_{0}\right\|_{3 / 2, \infty, D_{\rho}}\right) \\
& \quad \leq C\left(\left\|\phi_{1} f\right\|_{\dot{W}_{3 / 2, \infty}^{-1}\left(\boldsymbol{R}^{3}\right)}+\left\|\phi_{2} f\right\|_{\dot{W}_{3 / 2, \infty}^{-1}\left(D_{\rho}\right)}\right) .
\end{aligned}
$$

This together with (5.9) and (5.10) implies the estimate of $R f$ in $\dot{W}_{3 / 2, \infty}^{-1}(D)$ in (5.15).

Since the support of $R f$ is contained in $A_{\rho}$ we get as in the first part of the proof the estimate of $R f$ in $L_{3 / 2, \infty}\left(A_{\rho}\right)$.

By Lemma 5.1 and Proposition 5.3, we find that $(v, \pi)=(S f, \Pi f)$ is a $(3 / 2, \infty)$-weak solution of $(5.13)$.

Proposition 5.4. The operator $R$ is compact from $\dot{W}_{3 / 2, \infty}^{-1}(D)$ into itself, and $1+R$ has a bounded inverse in $\dot{W}_{3 / 2, \infty}^{-1}(D)$.

Proof. By Lemma 2.1 and (5.15) together with supp $R f \subset A_{\rho}$, the operator $R$ is compact in $\dot{W}_{3 / 2, \infty}^{-1}(D)$. To complete the proof, owing to the Fredholm theorem, it suffices to show that $1+R$ is injective in $\dot{W}_{3 / 2, \infty}^{-1}(D)$. Suppose that $(1+R) f=0$. Then, supp $f=\operatorname{supp} R f \subset A_{\rho}$. Also, in view of (5.13), the pair $(v, \pi)=(S f, \Pi f)$ for such $f$ is a $(3 / 2, \infty)$-weak solution to 


$$
L v+\nabla \pi=0, \quad \operatorname{div} v=0 \quad \text { in } D ;\left.\quad v\right|_{\partial D}=0 .
$$

From Proposition 5.1 it follows that $(v, \pi)=(0,0)$, which yields

$$
\left(S_{\infty} f, \Pi_{\infty} f\right)=(0,0), \quad|x| \geq \rho-1 ; \quad\left(S_{0} f, \Pi_{0} f\right)=(0,0), \quad x \in D_{\rho-4} .
$$

Both $\left(S_{\infty} f, \Pi_{\infty} f\right)$ and $\left(S_{0} f, \Pi_{0} f\right)$ are of class $\dot{W}_{3 / 2, \infty}^{1}\left(B_{\rho}\right) \times L_{3 / 2, \infty}\left(B_{\rho}\right)$ and they are $(3 / 2, \infty)$-weak solutions to

$$
L u+\nabla p=f, \quad \operatorname{div} u=0 \quad \text { in } B_{\rho} ;\left.\quad u\right|_{\partial B_{\rho}}=0,
$$

where now $\left(S_{0} f, \Pi_{0} f\right)$ is understood as its extension by zero on the region $B_{\rho} \backslash D_{\rho} \equiv \boldsymbol{R}^{3} \backslash D$. By the uniqueness of solutions on $B_{\rho}$, see Proposition 4.1, we obtain $\left(S_{\infty} f, \Pi_{\infty} f\right)=\left(S_{0} f, \Pi_{0} f\right)$ in $B_{\rho}$, which implies

$$
S_{\infty} f=S f=v=0, \quad \Pi_{\infty} f=\Pi f=\pi=0 \quad \text { in } B_{\rho} .
$$

Returning to the equation in the whole space $\boldsymbol{R}^{3}$, we obtain $f=0$ in $D$. This completes the proof.

Proof of Theorem 5.1. Since uniqueness follows from Proposition 5.1, we will show the existence of solutions together with (5.2).

(i) The case $(q, r)=(3 / 2, \infty)$ : By Propositions 5.3 and 5.4 the pair of

$$
u=T f:=S(1+R)^{-1} f, \quad p=Q f:=\Pi(1+R)^{-1} f,
$$

provides a $(3 / 2, \infty)$-weak solution of $(5.1)$ with $f \in \dot{W}_{3 / 2, \infty}^{-1}(D)$ and the estimate (5.2) holds.

We show that the constant $C>0$ in (5.2) is independent of $|\omega| \in[0, M]$, $M>0$. Suppose the contrary. Then there are sequences $\left(\omega_{n}\right)$ with $\left|\omega_{n}\right| \in[0, M]$ and $\left(f_{n}\right) \subset \dot{W}_{3 / 2, \infty}^{-1}(D)$ such that

$$
\begin{gathered}
\lim _{n \rightarrow \infty}\left\|f_{n}\right\|_{\dot{W}_{3 / 2, \infty}^{-1}(D)}=0, \\
\left\|\nabla u_{n}\right\|_{3 / 2, \infty}+\left\|p_{n}\right\|_{3 / 2, \infty}+\left\|\left(\omega_{n} \wedge x\right) \cdot \nabla u_{n}-\omega_{n} \wedge u_{n}\right\|_{\dot{W}_{3 / 2, \infty}^{-1}(D)}=1,
\end{gathered}
$$

where $\left(u_{n}, p_{n}\right) \in \dot{W}_{3 / 2, \infty}^{1}(D) \times L_{3 / 2, \infty}(D)$ is the corresponding weak solution. Hence there are subsequences, which we denote by the same symbols, so that

$$
w^{*}-\lim _{n \rightarrow \infty}\left(u_{n}, p_{n}\right)=(u, p) \quad \text { in } \dot{W}_{3 / 2, \infty}^{1}(D) \times L_{3 / 2, \infty}(D), \quad \lim _{n \rightarrow \infty} \omega_{n}=\alpha e_{3} .
$$

We set

$$
W_{3 / 2, \infty}^{1}\left(D_{\rho}\right)=\left\{u \in L_{3 / 2, \infty}\left(D_{\rho}\right) ; \nabla u \in L_{3 / 2, \infty}\left(D_{\rho}\right)\right\} .
$$

Since this space is compactly embedded into $L_{3 / 2, \infty}\left(D_{\rho}\right)$ and so is $L_{3 / 2, \infty}\left(D_{\rho}\right)$ into $\dot{W}_{3 / 2, \infty}^{-1}\left(D_{\rho}\right)$, see Lemma 2.1 (i), we have 


$$
\lim _{n \rightarrow \infty}\left(u_{n}, p_{n}\right)=(u, p) \quad \text { in } L_{3 / 2, \infty}\left(D_{\rho}\right) \times \dot{W}_{3 / 2, \infty}^{-1}\left(D_{\rho}\right) .
$$

On the other hand, it is possible to derive the a priori estimate

$$
\begin{gathered}
\left\|\nabla u_{n}\right\|_{3 / 2, \infty}+\left\|p_{n}\right\|_{3 / 2, \infty}+\left\|\left(\omega_{n} \wedge x\right) \cdot \nabla u_{n}-\omega_{n} \wedge u_{n}\right\|_{\dot{W}_{3 / 2, \infty}^{-1}(D)} \\
\leq C\left\{\left\|f_{n}\right\|_{\dot{W}_{3 / 2, \infty}^{-1}(D)}+\left(1+\left|\omega_{n}\right|\right)\left\|u_{n}\right\|_{3 / 2, \infty, D_{\rho}}\right. \\
\left.+\left\|p_{n}\right\|_{\dot{W}_{3 / 2, \infty}^{-1}\left(D_{\rho}\right)}+\left|\int_{D_{\rho}} \phi_{0}(x) p_{n}(x) d x\right|\right\}
\end{gathered}
$$

for the weak solutions $\left(u_{n}, p_{n}\right) \in \dot{W}_{3 / 2, \infty}^{1}(D) \times L_{3 / 2, \infty}(D)$, where the cut-off function $\phi_{0}$ is as in (5.5) and $C>0$ is independent of $\left|\omega_{n}\right|$. In fact, using Proposition 3.2, Lemma 4.1 and (2.8), we follow the cut-off argument in the proof of [28, Lemma 5.2] to obtain (5.19).

By (5.17), (5.18) and (5.19), we find, as $n \rightarrow \infty$,

$$
1 \leq C\left\{(1+\alpha)\|u\|_{3 / 2, \infty, D_{\rho}}+\|p\|_{\dot{W}_{3 / 2, \infty}^{-1}\left(D_{\rho}\right)}+\left|\int_{D_{\rho}} \phi_{0}(x) p(x) d x\right|\right\} .
$$

However, the limit $(u, p)$ is a $(3 / 2, \infty)$-weak solution to $(5.1)$ with angular velocity $\omega=\alpha e_{3}$ and $f=0$, so that Proposition 5.1 implies $(u, p)=(0,0)$, contradicting (5.20).

(ii) The case $3 / 2<q<3,1 \leq r \leq \infty$ : From [28] it follows that, given $f \in \dot{W}_{q}^{-1}(D), 3 / 2<q<3$, there is a unique $q$-weak solution

$$
u=T f \in \dot{W}_{q}^{1}(D), \quad p=Q f \in L_{q}(D)
$$

to (5.1) with bounded linear operators $(T, Q): \dot{W}_{q}^{-1}(D) \rightarrow \dot{W}_{q}^{1}(D) \times L_{q}(D)$. By real interpolation it is extended as a bounded operator from $\dot{W}_{q, r}^{-1}(D)$ to $\dot{W}_{q, r}^{1}(D) \times L_{q, r}(D)$ for every $r \in[1, \infty]$. Then $(u, p)=(T f, Q f)$ provides a $(q, r)$-weak solution. The assertion about the constant $C>0$ in (5.2) is proved in the same way as in the proof of the case $(q, r)=(3 / 2, \infty)$.

(iii) The case $(q, r)=(3,1)$ : Differently from the case $(q, r)=(3 / 2, \infty)$, the continuity argument as in [28] now works since $\left\{\operatorname{div} F ; F \in C_{0}^{\infty}(D)\right\}$ is dense in $\dot{W}_{3,1}^{-1}(D)$. For an external force in this dense subspace, we know from [28, Lemma 5.3] the existence of a distribution solution of class $(u, p) \in \dot{W}_{q}^{1}(D) \times$ $L_{q}(D)$ for all $q \in(3 / 2, \infty)$, which yields $(u, p) \in \dot{W}_{3,1}^{1}(D) \times L_{3,1}(D)$. On the other hand, as in (5.19) above, we follow again the cut-off argument in [28, Lemma 5.2] to find the a priori estimate 


$$
\begin{aligned}
&\|\nabla u\|_{3,1}+\|p\|_{3,1}+\|(\omega \wedge x) \cdot \nabla u-\omega \wedge u\|_{\dot{W}_{3,1}^{-1}(D)} \\
& \leq C\left\{\|f\|_{\dot{W}_{3,1}^{-1}(D)}+(1+|\omega|)\|u\|_{3,1, D_{\rho}}\right. \\
&\left.+\|p\|_{\dot{W}_{3,1}^{-1}\left(D_{\rho}\right)}+\left|\int_{D_{\rho}} \phi_{0}(x) p(x) d x\right|\right\}
\end{aligned}
$$

for $(3,1)$-weak solutions $(u, p)$. Since we have the uniqueness by Proposition 5.1 , we employ the same contradiction argument with use of (5.21) as in the above proof of the case $(q, r)=(3 / 2, \infty)$ to show the estimate $(5.2)$ with $(q, r)=$ $(3,1)$, which is independent of $|\omega| \in[0, M]$, for every $(3,1)$-weak solution $(u, p)$. This a priori estimate combined with the solvability for $f=\operatorname{div} F, F \in C_{0}^{\infty}(D)$, explained above easily gives the existence of a $(3,1)$-weak solution for general $f \in \dot{W}_{3,1}^{-1}(D)$ by continuity.

Finally, to prove Theorem 5.2, we will construct the solution of (5.1) when $(q, r)$ satisfies (1.12). Given $f \in \dot{W}_{q, r}^{-1}(D)$, there is $F \in L_{q, r}(D)$ such that $f=\operatorname{div} F$, see Lemma 2.2. We use the functions $\phi_{j}$ given by (5.5) and easily see that

$$
\operatorname{div}\left(\phi_{1} F\right) \in \dot{W}_{q, r}^{-1}\left(\boldsymbol{R}^{3}\right), \quad \operatorname{div}\left(\phi_{2} F\right) \in \dot{W}_{q, r}^{-1}\left(D_{\rho}\right)
$$

with

$$
\left\|\operatorname{div}\left(\phi_{1} F\right)\right\|_{\dot{W}_{q, r}^{-1}\left(\boldsymbol{R}^{3}\right)}+\left\|\operatorname{div}\left(\phi_{2} F\right)\right\|_{\dot{W}_{q, r}^{-1}\left(D_{\rho}\right)} \leq C\|f\|_{\dot{W}_{q, r}^{-1}(D)} .
$$

Note that the idea to use $\phi_{j} f$ does not work here, differently from the case $(q, r)=(3 / 2, \infty)$, see (5.9), (5.10). But, similarly to the construction of the solution for the case $(q, r)=(3 / 2, \infty)$, we take the solutions $\left(u_{\infty}, p_{\infty}\right)$ and $\left(u_{0}, p_{0}\right)$ obtained, respectively, in Proposition 3.2 with $g=0$ for the external force $\operatorname{div}\left(\phi_{1} F\right)$, where $u_{\infty}$ is chosen in such a way that $u_{\infty} \in L_{q_{*}, r}\left(\boldsymbol{R}^{3}\right)$, and in Proposition 4.1 for the external force $\operatorname{div}\left(\phi_{2} F\right)$, where $p_{0}$ is taken so that $\int_{D_{\rho}} p_{0}(x) d x=0$. We define the solution operators

$$
\begin{gathered}
\left(S_{\infty}, \Pi_{\infty}\right): \dot{W}_{q, r}^{-1}(D) \ni f \mapsto\left(u_{\infty}, p_{\infty}\right) \in \dot{W}_{q, r}^{1}\left(\boldsymbol{R}^{3}\right) \times L_{q, r}\left(\boldsymbol{R}^{3}\right), \\
\left(S_{0}, \Pi_{0}\right): \dot{W}_{q, r}^{-1}(D) \ni f \mapsto\left(u_{0}, p_{0}\right) \in \dot{W}_{q, r}^{1}\left(D_{\rho}\right) \times L_{q, r}\left(D_{\rho}\right) .
\end{gathered}
$$

As the first approximation of the solution for (5.1), one may take $(S f, \Pi f)$ as in (5.11). The same argument as in Proposition 5.3 together with (5.22) implies that

$$
(S f, \Pi f) \in \dot{W}_{q, r}^{1}(D) \times L_{q, r}(D)
$$

subject to the estimate 


$$
\|\nabla S f\|_{q, r}+\|\Pi f\|_{q, r} \leq C\|f\|_{\dot{W}_{q, r}^{-1}(D)}
$$

for some $C=C(q, r, M)>0$ uniformly in $|\omega| \in[0, M], M>0$.

We set $(v, \pi)=(S f, \Pi f)$, which solves the problem (5.13) with the remainder term $R f$ given by (5.14). Following [35], we intend to seek a solution $(u, p)$ to $(5.1)$ of the form

$$
u=v+\tilde{v}, \quad p=\pi+\tilde{\pi} .
$$

Then the second part $(\tilde{v}, \tilde{\pi})$ has to obey

$$
L \tilde{v}+\nabla \tilde{\pi}=-R f, \quad \operatorname{div} \tilde{v}=0 \quad \text { in } D ;\left.\quad \tilde{v}\right|_{\partial D}=0 .
$$

Now, instead of Lemma 5.1, we see that $R f \in L_{q, r}\left(A_{\rho}\right)$ with supp $R f \subset A_{\rho}$ subject to

$$
\|R f\|_{q, r, A_{\rho}} \leq C\|f\|_{\dot{W}_{q, r}^{-1}(D)},
$$

for some $C=C(q, r, M)>0$ uniformly in $|\omega| \in[0, M], M>0$. Unlike the cases (1.11), however, (5.25) never implies $R f \in \dot{W}_{q, r}^{-1}(D)$.

We are now in a position to complete the proof.

Proof of Theorem 5.2. For all $\psi \in C_{0}^{\infty}(D)$, we have by (5.25)

$$
|\langle R f, \psi\rangle| \leq\|R f\|_{q, r, A_{\rho}}\|\psi\|_{q^{\prime}, r^{\prime}, A_{\rho}} \leq C\|f\|_{\dot{W}_{q, r}^{-1}(D)}\|\psi\|_{\infty} .
$$

Thanks to (2.8), we find that $R f \in \dot{W}_{3 / 2, \infty}^{-1}(D)$ with

$$
\|R f\|_{\dot{W}_{3 / 2, \infty}^{-1}(D)} \leq C\|f\|_{\dot{W}_{q, r}^{-1}(D)},
$$

for some $C=C(q, r, M)>0$ uniformly in $|\omega| \in[0, M], M>0$. Hence, Theorem 5.1 for the case $(q, r)=(3 / 2, \infty)$ shows that the problem $(5.24)$ possesses a unique $(3 / 2, \infty)$-weak solution

$$
\tilde{v}=-T(R f) \in \dot{W}_{3 / 2, \infty}^{1}(D), \quad \tilde{\pi}=-Q(R f) \in L_{3 / 2, \infty}(D),
$$

where $(T, Q)$ is the solution operator for the problem (5.1), see (5.16). Then $(u, p)=(v+\tilde{v}, \pi+\tilde{\pi})$ actually provides a weak solution of class (5.3); its uniqueness follows from Proposition 5.2. We collect (5.23) for $(v, \pi)=$ $(S f, \Pi f),(5.2)$ with $(q, r)=(3 / 2, \infty)$ for $(\tilde{v}, \tilde{\pi})$ and (5.26) to obtain (5.4), with a constant $C>0$ independent of $|\omega| \in[0, M]$ for each $M>0$.

\section{Navier-Stokes problem}

This section is devoted to the proof of Theorem 1.1. We begin with the assertion on uniqueness. 
Proposition 6.1. For each $M>0$ there is a constant $\tilde{\eta}=\tilde{\eta}(M)>0$ such that for $|\omega| \in[0, M]$ a weak solution of (1.2), (1.3) in the class

$$
(\nabla u, p) \in L_{3 / 2, \infty}(D), \quad u \in L_{3, \infty}(D), \quad\|u\|_{3, \infty} \leq \tilde{\eta},
$$

is unique.

Proof. Let $(u, p)$ and $(\tilde{u}, \tilde{p})$ be solutions of (1.2), (1.3) in the class (6.1). Set $(w, \pi)=(u-\tilde{u}, p-\tilde{p})$, which obeys

$$
\begin{cases}L w+\nabla \pi+w \cdot \nabla u+\tilde{u} \cdot \nabla w=0 & \text { in } D, \\ \operatorname{div} w=0 & \text { in } D, \\ w=0 & \text { on } \partial D, \\ w \rightarrow 0 & \text { as }|x| \rightarrow \infty .\end{cases}
$$

Since $w=0$ on $\partial D$, it follows from (2.6) that $w \in \dot{W}_{3 / 2, \infty}^{1}(D)$. By Theorem 5.1, the weak Hölder inequality ([5, Lemma 2.1]) and (2.7), we see that

$$
\begin{aligned}
\|\nabla w\|_{3 / 2, \infty}+\|\pi\|_{3 / 2, \infty} & \leq C\|w \cdot \nabla u+\tilde{u} \cdot \nabla w\|_{\dot{W}_{3 / 2, \infty}^{-1}(D)} \\
& \leq C\left(\|u\|_{3, \infty}+\|\tilde{u}\|_{3, \infty}\right)\|w\|_{3, \infty} \\
& \leq \tilde{c}\left(\|u\|_{3, \infty}+\|\tilde{u}\|_{3, \infty}\right)\|\nabla w\|_{3 / 2, \infty} .
\end{aligned}
$$

One may take $\tilde{\eta}<1 /(2 \tilde{c})$, so that the condition (6.1) yields $w=0$ in $\dot{W}_{3 / 2, \infty}^{1}(D)$, and thus $\pi=0$ in $L_{3 / 2, \infty}(D)$.

Let

$$
T: \dot{W}_{3 / 2, \infty}^{-1}(D) \ni f \mapsto u \in \dot{W}_{3 / 2, \infty}^{1}(D)
$$

be the solution operator defined by Theorem 5.1 for the linearized problem (5.1), see (5.16). By (5.2) we have

$$
\|\nabla T f\|_{3 / 2, \infty} \leq C\|f\|_{\dot{W}_{3 / 2, \infty}^{-1}(D)} .
$$

Given $v \in \dot{W}_{3 / 2, \infty}^{1}(D)$ and $b$ defined by (2.11), we have

$$
\|(v+b) \otimes(v+b)\|_{3 / 2, \infty} \leq\|v+b\|_{3, \infty}^{2} \leq C\left(\|\nabla v\|_{3 / 2, \infty}+|\omega|\right)^{2}
$$

by (2.7). Since $b \in C_{0}^{\infty}\left(\boldsymbol{R}^{3}\right)$, we also have

$$
\|\nabla b+(\omega \wedge x) \otimes b-b \otimes(\omega \wedge x)\|_{3 / 2, \infty}=C\left(|\omega|+|\omega|^{2}\right) .
$$

Thus, $\Phi(v, b) \in \dot{W}_{3 / 2, \infty}^{-1}(D)$ satisfying

$$
\|\Phi(v, b)\|_{\dot{W}_{3 / 2, \infty}^{-1}(D)} \leq C\|\nabla v\|_{3 / 2, \infty}^{2}+C\left(|\omega|+|\omega|^{2}\right) .
$$

Therefore, given $f \in \dot{W}_{3 / 2, \infty}^{-1}(D)$, we define the operator $S$ from $\dot{W}_{3 / 2, \infty}^{1}(D)$ into itself by 


$$
S v=T[f-\Phi(v, b)]
$$

so that problem (2.12) is reduced to the fixed point problem

$$
v=S v \quad \text { in } \dot{W}_{3 / 2, \infty}^{1}(D) .
$$

Once we find a fixed point $v$ of the operator $S$, we obtain the associated pressure $p$ as well by Theorem 5.1 with $f$ replaced by $f-\Phi(v, b)$, and the pair $(v, p)$ is actually a weak solution to (2.12).

Proof of Theorem 1.1. It follows from (6.2) and (6.3) that

$$
\begin{aligned}
\|\nabla S v\|_{3 / 2, \infty} & =\|\nabla T[f-\Phi(v, b)]\|_{3 / 2, \infty} \\
& \leq c_{0}\left(\|f\|_{\dot{W}_{3 / 2, \infty}^{-1}(D)}+\|\nabla v\|_{3 / 2, \infty}^{2}+|\omega|^{2}+|\omega|\right) .
\end{aligned}
$$

Put $\rho=2 c_{0}\left(\|f\|_{\dot{W}_{3 / 2, \infty}^{-1}(D)}+|\omega|^{2}+|\omega|\right)$ and define the closed ball

$$
\mathscr{B}_{\rho}=\left\{v \in \dot{W}_{3 / 2, \infty}^{1}(D) ;\|\nabla v\|_{3 / 2, \infty} \leq \rho\right\}
$$

in $\dot{W}_{3 / 2, \infty}^{1}(D)$. Assume that

$$
\|f\|_{\dot{W}_{3 / 2, \infty}^{-1}(D)}+|\omega|^{2}+|\omega| \leq \frac{1}{8 c_{0}^{2}},
$$

or equivalently, $\rho \leq 1 / 4 c_{0}$. Then we see by (6.5) that $v \in \mathscr{B}_{\rho}$ implies

$$
\|\nabla S v\|_{3 / 2, \infty} \leq c_{0} \rho^{2}+\frac{\rho}{2} \leq \rho .
$$

Similarly, we have

$$
\begin{aligned}
\|\nabla(S v-S w)\|_{3 / 2, \infty} & =\|\nabla T[\Phi(v, b)-\Phi(w, b)]\|_{3 / 2, \infty} \\
& \leq C\|\Phi(v, b)-\Phi(w, b)\|_{\dot{W}_{3 / 2, \infty}^{-1}(D)} \\
& \leq C\|(v-w) \otimes(v+b)+(w+b) \otimes(v-w)\|_{3 / 2, \infty} \\
& \leq c_{0}\left(\|\nabla v\|_{3 / 2, \infty}+\|\nabla w\|_{3 / 2, \infty}+|\omega|\right)\|\nabla(v-w)\|_{3 / 2, \infty} \\
& \leq c_{0}(2 \rho+|\omega|)\|\nabla(v-w)\|_{3 / 2, \infty}
\end{aligned}
$$

for $v, w \in \mathscr{B}_{\rho}$, where $c_{0}>0$ is the same constant as in (6.5). By (6.6) we find $c_{0}(2 \rho+|\omega|)<1$, so that $S$ is contractive from $\mathscr{B}_{\rho}$ into itself. We thus obtain a solution by Banach's fixed point theorem. By Proposition 6.1, the obtained solution $(u, p)=(v+b, p)$ provides a unique solution of (1.2), (1.3) in the class (6.1) as long as $\|v+b\|_{3, \infty} \leq \tilde{\eta}$. 
We conclude this paper with a further regularity result of the solution obtained in Theorem 1.1 under an additional assumption on the external force. This result is actually used in the study of the stability of the stationary solution, see Hishida and Shibata [29, Section 9].

Theorem 6.1. Let $3 / 2<q<3$. Then there is a constant $\eta^{\prime}=\eta^{\prime}(q, D) \in$ $(0, \eta]$ such that for

$$
f \in \dot{W}_{3 / 2, \infty}^{-1}(D) \cap \dot{W}_{q, \infty}^{-1}(D)
$$

with

$$
|\omega|+\|f\|_{\dot{W}_{3 / 2, \infty}^{-1}(D)} \leq \eta^{\prime}
$$

the solution $(u, p)$ obtained in Theorem 1.1 for the problem (1.2), (1.3) enjoys

$$
\nabla u, p \in L_{3 / 2, \infty}(D) \cap L_{q, \infty}(D)
$$

subject to the estimate

$$
\begin{gathered}
\|(\nabla u, p)\|_{3 / 2, \infty}+\|(\nabla u, p)\|_{q, \infty}+\|u\|_{3, \infty}+\|u\|_{q_{*}, \infty} \\
\leq C\left(|\omega|+\|f\|_{\dot{W}_{3 / 2, \infty}^{-1}(D)}+\|f\|_{\dot{W}_{q, \infty}^{-1}(D)}\right),
\end{gathered}
$$

with some $C=C(q)>0$ independent of $|\omega|$ and $f$, where $1 / q_{*}=1 / q-1 / 3$.

Theorem 6.1 is a generalization of a result of Kozono and Yamazaki [32, Main Theorem (3)] for the case $\omega=0$, but the proof below is somewhat different from theirs.

Fix $q \in(3 / 2,3)$ and assume that $f \in \dot{W}_{3 / 2, \infty}^{-1}(D) \cap \dot{W}_{q, \infty}^{-1}(D)$ satisfies (1.5). Let $(v, p)=(u-b, p) \in \dot{W}_{3 / 2, \infty}^{1}(D) \times L_{3 / 2, \infty}(D)$ be the unique solution to the reduced problem (2.12) obtained in Theorem 1.1. Using the solution operator $T$ defined by Theorem 5.1 for the linearized problem (5.1), we define an auxiliary operator $A_{v}$ by

$$
A_{v} w=T[f-\operatorname{div}\{v \otimes w+H(v, b)\}],
$$

where

$$
H(v, b)=v \otimes b+b \otimes v+b \otimes b-\nabla b-(\omega \wedge x) \otimes b+b \otimes(\omega \wedge x)
$$

so that $\Phi(v, b)=\operatorname{div}[v \otimes v+H(v, b)]$, see (2.13).

Given $w \in \dot{W}_{3 / 2, \infty}^{1}(D) \cap \dot{W}_{q, \infty}^{1}(D)$, we see from (2.7) that

$$
\begin{aligned}
\|v \otimes w\|_{3 / 2, \infty}+\|v \otimes w\|_{q, \infty} & \leq\|v\|_{3, \infty}\left(\|w\|_{3, \infty}+\|w\|_{q_{*}, \infty}\right) \\
& \leq C\|v\|_{3, \infty}\left(\|\nabla w\|_{3 / 2, \infty}+\|\nabla w\|_{q, \infty}\right)
\end{aligned}
$$

and that 


$$
\|H(v, b)\|_{3 / 2, \infty}+\|H(v, b)\|_{q, \infty} \leq C|\omega| \cdot\|v\|_{3, \infty}+C\left(|\omega|^{2}+|\omega|\right) .
$$

Hence, by Theorem 5.1 one can regard $A_{v}$ as an operator from $\dot{W}_{3 / 2, \infty}^{1}(D) \cap$ $\dot{W}_{q, \infty}^{1}(D)$ into itself. We thus consider the equation

$$
w=A_{v} w
$$

in $\dot{W}_{3 / 2, \infty}^{1}(D) \cap \dot{W}_{q, \infty}^{1}(D)$.

We have the following uniqueness assertion for (6.11).

Proposition 6.2. For each $M>0$, let $\tilde{\eta}=\tilde{\eta}(M)>0$ be the same constant as in Proposition 6.1. For $|\omega| \in[0, M]$ the solution of (6.11) in the class $w \in \dot{W}_{3 / 2, \infty}^{1}(D)$ is unique provided that $\|v\|_{3, \infty} \leq \tilde{\eta}$.

Proof. Let $w_{1}, w_{2} \in \dot{W}_{3 / 2, \infty}^{1}(D)$ be solutions of (6.11) and set $w=w_{1}-w_{2}$. Similarly to the proof of Proposition 6.1, we obtain by (6.2) and (2.7)

$$
\begin{aligned}
\|\nabla w\|_{3 / 2, \infty} & =\left\|\nabla\left(A_{v} w_{1}-A_{v} w_{2}\right)\right\|_{3 / 2, \infty}=\|\nabla T[\operatorname{div}(v \otimes w)]\|_{3 / 2, \infty} \\
& \leq \tilde{c}\|v\|_{3, \infty}\|\nabla w\|_{3 / 2, \infty} \leq \frac{1}{2}\|\nabla w\|_{3 / 2, \infty},
\end{aligned}
$$

provided that $\|v\|_{3, \infty} \leq \tilde{\eta}$, since $\tilde{c}$ is the same constant as in the proof of Proposition 6.1. This implies $w=0$ in $\dot{W}_{3 / 2, \infty}^{1}(D)$.

In view of (6.4) and (6.8), we have

$$
A_{v} v=T[f-\Phi(v, b)]=S v=v
$$

and, therefore, $v$ itself satisfies $(6.11)$ in $\dot{W}_{3 / 2, \infty}^{1}(D)$. Thus, it suffices to find a solution $w \in \dot{W}_{3 / 2, \infty}^{1}(D) \cap \dot{W}_{q, \infty}^{1}(D)$ of $(6.11)$; then, by Proposition 6.2,w must coincide with $v$. As a consequence, $v \in \dot{W}_{q, \infty}^{1}(D)$.

Proof of Theorem 6.1. By virtue of (6.9) one can define the linear operator $G_{v}$ from $\dot{W}_{3 / 2, \infty}^{1}(D) \cap \dot{W}_{q, \infty}^{1}(D)$ into itself by

$$
G_{v} w=T[\operatorname{div}(v \otimes w)]
$$

to rewrite the equation (6.11) as

$$
w+G_{v} w=T[f-\operatorname{div} H(v, b)] .
$$

It follows from (5.2) together with (6.9) that

$$
\left\|\nabla G_{v} w\right\|_{3 / 2, \infty}+\left\|\nabla G_{v} w\right\|_{q, \infty} \leq \tilde{c}_{q}\|v\|_{3, \infty}\left(\|\nabla w\|_{3 / 2, \infty}+\|\nabla w\|_{q, \infty}\right)
$$

for $w \in \dot{W}_{3 / 2, \infty}^{1}(D) \cap \dot{W}_{q, \infty}^{1}(D)$. If

$$
\|v\|_{3, \infty} \leq \frac{1}{2 \tilde{c}_{q}}
$$


then there is a bounded inverse $\left(1+G_{v}\right)^{-1}=\sum_{n=0}^{\infty}\left(-G_{v}\right)^{n}$ as Neumann series in $\dot{W}_{3 / 2, \infty}^{1}(D) \cap \dot{W}_{q, \infty}^{1}(D)$, so that the equation (6.11) possesses a solution

$$
w=\left(1+G_{v}\right)^{-1} T[f-\operatorname{div} H(v, b)] \in \dot{W}_{3 / 2, \infty}^{1}(D) \cap \dot{W}_{q, \infty}^{1}(D)
$$

with

$$
\begin{aligned}
\|\nabla w\|_{q, \infty} & \leq 2\|\nabla T[f-\operatorname{div} H(v, b)]\|_{q, \infty} \\
& \leq C\left(\|f\|_{\dot{W}_{q, \infty}^{-1}(D)}+\|v\|_{3, \infty}^{2}+|\omega|^{2}+|\omega|\right),
\end{aligned}
$$

which follows from (5.2) and (6.10). Hence, under the condition $\|v\|_{3, \infty} \leq \tilde{\eta}$ as well as $(6.12)$, we are led to $v=w \in \dot{W}_{q, \infty}^{1}(D)$ by Proposition 6.2 , that together with (2.7) yields

$$
\|\nabla v\|_{q, \infty}+\|v\|_{q_{*}, \infty} \leq C\left(\|f\|_{\dot{W}_{q, \infty}^{-1}(D)}+\|v\|_{3, \infty}^{2}+|\omega|^{2}+|\omega|\right) .
$$

We go back to (2.12) and use Theorem 5.1 again to see that $p \in L_{q, \infty}(D)$ subject to the estimate

$$
\begin{aligned}
\|p\|_{q, \infty} & \leq C\|f-\Phi(v, b)\|_{\dot{W}_{q, \infty}^{-1}(D)} \\
& \leq C\left(\|f\|_{\dot{W}_{q_{, \infty}}^{-1}(D)}+\|v\|_{3, \infty}\|v\|_{q_{*}, \infty}+\|v\|_{3, \infty}^{2}+|\omega|^{2}+|\omega|\right) .
\end{aligned}
$$

This combined with (6.13) implies the estimate of $(u, p)=(v+b, p)$ in (6.7), which completes the proof.

Acknowledgement. The second author would like to thank Prof. Y. Shibata for showing [35] before publication with valuable discussions about it. He is supported in part by Grand-in-Aid for Scientific Research, No. 16540143, from the Japan Society for the Promotion of Science. The authors are grateful to the referee for helpful suggestion.

\section{References}

[1] Bergh, J. and Löfström, J., Interpolation Spaces, Springer, Berlin, 1976.

[2] Bogovskiǐ, M. E., Solution of the first boundary value problem for the equation of continuity of an incompressible medium, Soviet Math. Dokl., 20 (1979), 1094-1098.

[3] Borchers, W., Zur Stabilität und Faktorisierungsmethode für die Navier-Stokes-Gleichungen inkompressibler viskoser Flüssigkeiten, Habilitationsschrift, Universität Paderborn, 1992.

[4] Borchers, W. and Miyakawa, T., Algebraic $L^{2}$ decay for Navier-Stokes flows in exterior domains, Acta Math., 165 (1990), 189-227.

[ 5 ] Borchers, W. and Miyakawa, T., On stability of exterior stationary Navier-Stokes flows, Acta Math., 174 (1995), 311-382.

[6] Borchers, W. and Sohr, H., On the equations $\operatorname{rot} v=g$ and $\operatorname{div} u=f$ with zero boundary conditions, Hokkaido Math. J., 19 (1990), 67-87. 
[7] Cattabriga, L., Su un problema al contorno relativo al sistema di equazioni di Stokes, Rend. Sem. Math. Univ. Padova, 31 (1961), 308-340.

[8] Chemin, J.-Y., Perfect Incompressible Fluids, Clarendon Press, Oxford 1998.

[9] Chen, Z. M. and Miyakawa, T., Decay properties of weak solutions to a perturbed NavierStokes system in $\boldsymbol{R}^{n}$, Adv. Math. Sci. Appl., 7 (1997), 741-770.

[10] Cumsille, P. and Tucsnak, T., Wellposedness for the Navier-Stokes flow in the exterior of a rotating obstacle, Math. Methods Appl. Sci., 29 (2006), 595-623.

[11] Farwig, R., The stationary Navier-Stokes equations in a 3D-exterior domain, Recent Topics on Mathematical Theory of Viscous Incompressible Fluid, 53-115, Lecture Notes in Num. Appl. Anal., 16, Kinokuniya, Tokyo, 1998.

[12] Farwig, R., An $L^{q}$-analysis of viscous fluid flow past a rotating obstacle, Tohoku Math. J., 58 (2006), 129-147.

[13] Farwig, R., Estimates of lower order derivatives of viscous fluid flow past a rotating obstacle, Banach Center Publications, 70, 73-84, Warsaw, 2005.

[14] Farwig, R., Hishida, T. and Müller, D., $L^{q}$-theory of a singular "winding" integral operator arising from fluid dynamics, Pacific. J. Math., 215 (2004), 297-312.

[15] Farwig, R., Krbec, M. and Nečasová, Š., A weighted $L^{q}$-approach to Stokes flow around a rotating body, Ann. Univ. Ferrara, Sez. VII (2007), to appear.

[16] Farwig, R. and Neustupa, J., On the spectrum of a Stokes-type operator arising from flow around a rotating body, Manuscripta Mathematica, 122 (2007), 419-437.

[17] Galdi, G. P., An Introduction to the Mathematical Theory of the Navier-Stokes Equations, Vol. I: Linearized Steady Problems, Vol. II: Nonlinear Steady Problems, revised edition, Springer, New York, 1998.

[18] Galdi, G. P., On the motion of a rigid body in a viscous liquid: a mathematical analysis with applications, Handbook of Mathematical Fluid Dynamics, Vol. I, 653-791, North-Holland, Amsterdam, 2002.

[19] Galdi, G. P., Steady flow of a Navier-Stokes fluid around a rotating obstacle, J. Elasticity, 71 (2003), 1-31.

[20] Galdi, G. P. and Silvestre, A. L., Strong solutions to the Navier-Stokes equations around a rotating obstacle, Arch. Rational Mech. Anal., 176 (2005), 331-350.

[21] Galdi, G. P. and Silvestre, A. L., The steady motion of a Navier-Stokes liquid around a rigid body, Arch. Rational Mech. Anal., 184 (2007), 371-400.

[22] Galdi, G. P. and Simader, C. G., Existence, uniqueness and $L^{q}$-estimates for the Stokes problem in exterior domains, Arch. Rational Mech. Anal., 112 (1990), 291318.

[23] Geissert, M., Heck, H. and Hieber, M., $L^{p}$-theory of the Navier-Stokes flow in the exterior of a moving or rotating obstacle, J. Reine Angew. Math., 596 (2006), 45-62.

[24] Hishida, T., The Stokes operator with rotation effect in exterior domains, Analysis, 19 (1999), 51-67.

[25] Hishida, T., An existence theorem for the Navier-Stokes flow in the exterior of a rotating obstacle, Arch. Rational Mech. Anal., 150 (1999), 307-348.

[26] Hishida, T., $L^{2}$ theory for the operator $\Delta+(k \times x) \cdot \nabla$ in exterior domains, Nihonkai Math. J., 11 (2000), 103-135.

[27] Hishida, T., On the Navier-Stokes flow around a rigid body with a prescribed rotation, Nonlinear Analysis, 47 (2001), 4217-4231.

[28] Hishida, T., $L^{q}$ estimates of weak solutions to the stationary Stokes equations around a rotating body, J. Math. Soc. Japan, 58 (2006), 743-767. 
[29] Hishida, T. and Shibata, Y., $\quad L_{p}-L_{q}$ estimate of the Stokes operator and Navier-Stokes flows in the exterior of a rotating obstacle, Arch. Rational Mech. Anal., to appear.

[30] Kozono, H. and Sohr, H., New a priori estimates for the Stokes equations in exterior domains, Indiana Univ. Math. J., 41 (1991), 1-27.

[31] Kozono, H. and Sohr, H., On a new class of generalized solutions for the Stokes equations in exterior domains, Ann. Scuola Norm. Sup. Pisa, 19 (1992), 155-181.

[32] Kozono, H. and Yamazaki, M., Exterior problem for the stationary Navier-Stokes equations in the Lorentz space, Math. Ann., 310 (1998), 279-305.

[33] Nečasová, Š., Asymptotic properties of the steady fall of a body in viscous fluid, Math. Methods Appl. Sci., 27 (2004), 1969-1995.

[34] Serre, D., Chute libre d'un solide dans un fluide visqueux incompressible. Existence, Japan J. Appl. Math., 4 (1987), 99-110.

[35] Shibata, Y. and Yamazaki, M., Uniform estimates in the velocity at infinity for stationary solutions to the Navier-Stokes exterior problem, Japanese J. Math., 31 (2005), 225-279.

[36] Silvestre, A. L., On the existence of steady flows of a Navier-Stokes liquid around a moving rigid body, Math. Methods Appl. Sci., 27 (2004), 1399-1409.

[37] Solonnikov, V. A., Estimates for solutions of nonstationary Navier-Stokes equations, J. Sov. Math., 8 (1977), 467-529.

\author{
nuna adreso: \\ Reinhard Farwig \\ Fachbereich Mathematik \\ Technische Universität Darmstadt \\ 64289 Darmstadt \\ Germany \\ E-mail: farwig@mathematik.tu-darmstadt.de \\ Toshiaki Hishida \\ Department of Applied Mathematics \\ Faculty of Engineering \\ Niigata University \\ Niigata 950-2181 \\ Japan \\ E-mail: hishida@eng.niigata-u.ac.jp
}

(Ricevita la 6-an de marto, 2006)

(Reviziita la 14-an de aprilo, 2007) 